\title{
Analysis and Application of Single Level, Multi-Level Monte Carlo and Quasi-Monte Carlo Finite Element Methods for Time-Dependent Maxwell's Equations with Random Inputs
}

\author{
Xiang Wang ${ }^{1}$, Jichun $\mathrm{Li}^{2, *}$ and Zhiwei Fang ${ }^{2}$ \\ ${ }^{1}$ Department of Mathematical Sciences, Nanchang University, Nanchang, China. \\ 2 Department of Mathematical Sciences, University of Nevada Las Vegas, Las Vegas, \\ Nevada 89154-4020, USA.
}

Received 15 January 2020; Accepted (in revised version) 4 May 2020

\begin{abstract}
This article is devoted to three quadrature methods for the rapid solution of stochastic time-dependent Maxwell's equations with uncertain permittivity, permeability and initial conditions. We develop the mathematical analysis of the error estimate for single level Monte Carlo method, multi-level Monte Carlo method, and the quasi-Monte Carlo method. The theoretical results are supplemented by numerical experiments.
\end{abstract}

AMS subject classifications: 65N30, 35L15, 78-08

Key words: Maxwell's equations, uncertainty quantification, edge element.

\section{Introduction}

Consider the Maxwell's equations with random coefficients which are parameterized by a random vector $\boldsymbol{y} \in \mathbb{R}^{n}$ :

$$
\begin{aligned}
& \epsilon(\boldsymbol{x}, \boldsymbol{y}) \partial_{t} \boldsymbol{E}(\boldsymbol{x}, t ; \boldsymbol{y})=\nabla \times \boldsymbol{H}(\boldsymbol{x}, t ; \boldsymbol{y}), \\
& \mu(\boldsymbol{x}, \boldsymbol{y}) \partial_{t} \boldsymbol{H}(\boldsymbol{x}, t ; \boldsymbol{y})=-\nabla \times \boldsymbol{E}(\boldsymbol{x}, t ; \boldsymbol{y}),
\end{aligned}
$$

where $\epsilon$ is the permittivity, $\mu$ is the permeability, and $E$ and $H$ represent the electric and magnetic fields, respectively. We assume that the spatial variable $x \in D \subseteq \mathbb{R}^{3}$, and the time variable $t \in(0, T]$. Here $D$ is a bounded Lipschitz polyhedral domain with connected

*Corresponding author. Email addresses: wangxiang49@ncu.edu.cn (X. Wang), jichun.li@unlv.edu (J. Li), fangz1@unlv.nevada.edu (Z. Fang) 
boundary $\partial D$. The curl operators are understood to operate on the spatial variables $x$, and the parameter vector $\boldsymbol{y}=\left(y_{1}, y_{2}, \cdots, y_{n}\right)=\left(y_{i}\right)_{i=1}^{n}$ consists of $n$ parameters $y_{i}$ which are assumed to be independent and identically distributed (i.i.d.) on [0,1], i.e.,

$$
y \in[0,1]^{n}:=U .
$$

The probability measure for $y$ is defined as $d y=\prod_{i=1}^{n} d y_{i}$.

To complete the problem, we assume that Eqs. (1.1)-(1.2) satisfy the perfect conducting (PEC) boundary condition:

$$
\mathbf{n} \times \boldsymbol{E}(\boldsymbol{x}, t ; \boldsymbol{y})=0 \quad \forall \boldsymbol{x} \in \partial D, \forall t \in(0, T] \text { and } \forall \boldsymbol{y} \in U,
$$

where $\mathbf{n}$ is the outward unit normal vector on $\partial D$. Furthermore, we assume that the Maxwell's equations (1.1)-(1.2) satisfy the following initial conditions:

$$
E(x, 0 ; y)=E_{0}(x, y), \quad H(x, 0 ; y)=H_{0}(x, y),
$$

where $E_{0}(x, y)$ and $H_{0}(x, y)$ are some given functions.

In the past two decades, the study of uncertainty quantification (UQ) got great attentions across different disciplines of sciences and engineering as detailed in recent review articles $[19,27,36,38]$ and monographs $[16,33,41,42,47,50]$. Uncertainty quantification plays an important part in electromagnetic material design. For example, [46] presented a computational stochastic methodology for generating and optimizing random metamaterial configurations. Compared to many excellent numerical analysis papers published for stochastic elliptic problems (e.g., [1,2,8,49]), stochastic parabolic equations [48] and stochastic hyperbolic or wave equations $[24,26,35,43,45,52]$, elastic waves scattering in random media $[3,15]$, stochastic porous media flow [13], radiative transfer equations with uncertain coefficients [51], the mathematical literature on UQ for Maxwell's equations is less developed. In 2006, Chauviere et al. [6] developed both the stochastic Galerkin method and stochastic collocation method for the time-dependent Maxwell's equations with uncertainties caused by the physical materials, the source wave and the physical domain. In 2015, Benner and Schneider [5] described several techniques for the time-harmonic Maxwell's equations by using stochastic collocation method. In 2016, Römer et al. [37] discussed a stochastic nonlinear magnetostatic problem solved by the stochastic collocation method. In 2018, Kamilis and Polydorides [25] considered an UQ problem for the low-frequency, time-harmonic Maxwell's equations with lognormal random conductivity. Also Jerez et al. [23] and Hao et al. [20] investigated the time-harmonic Maxwell's equations with random interfaces. In 2019, Chen et al. [7] analyzed a semiimplicit Euler scheme for discretizing the stochastic Maxwell's equations with multiplicative Itô noise, and derived the mean-square convergence. Recently, the authors carried out the error analysis of stochastic collocation method $[30,31]$ and stochastic Galerkin method [14] for time-dependent Maxwell's equations.

In this paper, the uncertain permittivity and permeability $\epsilon$ and $\mu$ in (1.1)-(1.2) are assumed to depend on both the spatial variable $x$ and the parameter $y$, and they are 
bounded below and above, i.e., there exist constants $\epsilon^{-}, \epsilon^{+}, \mu^{-}$and $\mu^{+}$such that the following inequalities

$$
0<\epsilon^{-} \leq \epsilon(\boldsymbol{x}, \boldsymbol{y}) \leq \epsilon^{+}<\infty, \quad 0<\mu^{-} \leq \mu(x, y) \leq \mu^{+}<\infty,
$$

hold true for any $x \in D$ and $y \in U$ almost surely (a.s.).

Our goal is to obtain statistical information on the solution $(\boldsymbol{E}, \boldsymbol{H})$ to (1.1)-(1.2), especially its expected value, which is defined on $U$ by

$$
\mathbb{E}[u]=\int_{U} u(\boldsymbol{y}) d \boldsymbol{y}, \quad \text { for } u=E, H .
$$

To approximate the expected value, we can adopt the single level Monte Carlo (SLMC), multi-level Monte Carlo (MLMC) quadrature rules (an excellent review is given by Giles [17]), and the Quasi-Monte Carlo (QMC) quadrature method. There is a huge list of the literature on the application of QMC to PDEs (especially elliptic PDEs) with random coefficients, see $[4,9,18,21]$ and references therein. To the best of our knowledge, there exist few work in the literature which study the QMC method for solving the Maxwell's equations with random inputs.

Since the solutions of (1.1)-(1.2) involve an extra parameter $y$, we introduce the following space to measure the solutions:

$$
L^{r}(U ; V)=\left\{v: U \mapsto V:\|v\|_{L^{r}(U ; V)}<\infty\right\},
$$

where $V$ is a Banach space of real-valued functions on domain $D$ with norm $\|\cdot\|_{V}$, and the space $L^{r}(U ; V)$ is equipped with the norm

$$
\|v\|_{L^{r}(U ; V)}:= \begin{cases}\left(\int_{U}\|v(\cdot, \boldsymbol{y})\|_{V}^{r} d \boldsymbol{y}\right)^{\frac{1}{r}} & \text { if } 0<r<\infty, \\ \operatorname{essup}_{\boldsymbol{y} \in U}\|v(\cdot, \boldsymbol{y})\|_{V} & \text { if } r=\infty .\end{cases}
$$

In this paper, to make notation clean, we use the following bold face norms to represent the corresponding norms for vectors:

$$
\boldsymbol{L}^{2}(D)=\left(L^{2}(D)\right)^{3}, \quad \boldsymbol{H}^{r}(D)=\left(H^{r}(D)\right)^{3}, \quad \boldsymbol{L}^{\infty}(D)=\left(L^{\infty}(D)\right)^{3} .
$$

We also adopt the following notations

$$
\|v\|_{L^{2}(D \times U)}:=\|v\|_{L^{2}\left(U ; \mathbf{L}^{2}(D)\right)^{\prime}} \quad\|v\|_{L^{\infty}(D \times U)}:=\operatorname{esssup}_{\boldsymbol{y} \in U}\|v(\cdot, \boldsymbol{y})\|_{L^{\infty}(D)},
$$

and denote the following Hilbert spaces:

$$
\begin{aligned}
& H(\operatorname{div} ; D)=\left\{\mathbf{u} \in L^{2}(D): \nabla \cdot \mathbf{u} \in L^{2}(D)\right\}, \\
& H(\operatorname{curl} ; D)=\left\{\mathbf{u} \in L^{2}(D): \nabla \times \mathbf{u} \in L^{2}(D)\right\}, \\
& H_{0}(\operatorname{curl} ; D)=\{\mathbf{u} \in H(\operatorname{curl} ; D): \mathbf{n} \times \mathbf{u}=0 \text { on } \partial D\} .
\end{aligned}
$$


The structure of this paper is as follows. Section 2 is dedicated to development and error analysis for the single level and multi-level Monte Carlo finite element methods. In Section 3, we introduce the QMC method, establish the regularity analysis of the solution with respect to the random vector, and prove the error estimate of the QMC method. Section 4 presents numerical results which confirm our theoretical results. We conclude the paper in Section 5.

\section{The Monte Carlo finite element methods}

\subsection{Mixed finite elements for Maxwell's equations}

The idea of Monte Carlo finite element method is very simple: given a sample $y,(2.1)$ (2.2) becomes a deterministic problem in the physical domain $D$, which can be solved by any classic finite element method for Maxwell's equations [32,34]. To solve the problem (2.1)-(2.2) by a finite element method, we partition the physical domain $D$ by a family of regular cubic or tetrahedral mesh $T_{h}$ with maximum mesh size $h$, and adopt the $r$-th $(r \geq 1$ order Raviart-Thomas-Nédélec (RTN) mixed finite element spaces $U_{h}$ and $V_{h}$ [32,34]: For any $r \geq 1$, on tetrahedral elements:

$$
\begin{aligned}
& \boldsymbol{u}_{h}=\left\{\boldsymbol{u}_{h} \in H(\operatorname{div} ; D)\left|\boldsymbol{u}_{h}\right|_{K} \in\left(p_{r-1}\right)^{3} \oplus \tilde{p}_{r-1} \boldsymbol{x}, \forall K \in T_{h}\right\}, \\
& \boldsymbol{V}_{h}=\left\{\boldsymbol{v}_{h} \in H(\operatorname{curl} ; D)\left|\boldsymbol{v}_{h}\right|_{K} \in\left(p_{r-1}\right)^{3} \oplus S_{r}, \forall K \in T_{h}\right\}, \quad S_{r}=\left\{\vec{p} \in\left(\tilde{p}_{r}\right)^{3}, \boldsymbol{x} \cdot \vec{p}=0\right\},
\end{aligned}
$$

or on cubic elements:

$$
\begin{aligned}
& \boldsymbol{u}_{h}=\left\{\boldsymbol{u}_{h} \in H(\text { div } ; D)\left|\boldsymbol{u}_{h}\right|_{K} \in Q_{r, r-1, r-1} \times Q_{r-1, r, r-1} \times Q_{r-1, r-1, r}, \quad \forall K \in T_{h}\right\}, \\
& \boldsymbol{V}_{h}=\left\{\boldsymbol{v}_{h} \in H(\text { curl } ; D)\left|\boldsymbol{v}_{h}\right|_{K} \in Q_{r-1, r, r} \times Q_{r, r-1, r} \times Q_{r, r, r-1}, \quad \forall K \in T_{h}\right\} .
\end{aligned}
$$

Here $p_{r-1}$ denotes the space of polynomials of degree $r-1, \tilde{p}_{r}$ denotes the space of homogeneous polynomials of degree $r$, and $Q_{i, j, k}$ denotes the space of polynomials whose degrees are less than or equal to $i, j, k$ in variables $x, y, z$, respectively. To impose the PEC boundary condition, we denote $\boldsymbol{V}_{h}^{0}=\left\{\boldsymbol{v} \in \boldsymbol{V}_{h}: \boldsymbol{v} \times \boldsymbol{n}=0\right.$ on $\left.\partial D\right\}$.

To define a fully discrete scheme, we divide the time interval $[0, T]$ into $M$ uniform subintervals by points $0=t_{0}<t_{1}<\cdots<t_{K}=T$, where $t_{k}=k \tau$, and $\tau=T / K$. Moreover, we denote the $k$-th subinterval by $I_{k}=\left[t_{k-1}, t_{k}\right]$, and the central difference and average operators for any time solutions $\mathbf{u}^{k}=\mathbf{u}(\cdot, k \tau)$ :

$$
\delta_{\tau} \mathbf{u}^{k-\frac{1}{2}}=\frac{\mathbf{u}^{k}-\mathbf{u}^{k-1}}{\tau}, \quad \overline{\mathbf{u}}^{k-\frac{1}{2}}=\frac{\mathbf{u}^{k}+\mathbf{u}^{k-1}}{2} .
$$

Before we construct the finite element scheme, we need to formulate a weak formulation for the model problem (1.1)-(1.2). For any fixed parameterized vector $y$, multiplying (1.1) and (1.2) by $\phi \in H_{0}($ curl; $D)$ and $\psi \in H($ div; $D)$, respectively, then using Green's theorem, 
we obtain

$$
\begin{aligned}
& \left(\epsilon \partial_{t} \boldsymbol{E}, \boldsymbol{\phi}\right)=(\boldsymbol{H}, \nabla \times \boldsymbol{\phi}), \\
& \left(\mu \partial_{t} \boldsymbol{H}, \boldsymbol{\psi}\right)=-(\nabla \times \boldsymbol{E}, \boldsymbol{\psi}),
\end{aligned}
$$

where $(\cdot, \cdot)$ denotes the usual inner product on $L^{2}(D)$.

Now we can formulate our Crank-Nicolson mixed finite element scheme: for $k=$ $1,2, \cdots K$, find $\boldsymbol{E}_{h}^{k} \in \mathbf{V}_{h}^{0}$ and $\boldsymbol{H}_{h}^{k} \in \mathbf{U}_{h}$ such that:

$$
\begin{array}{ll}
\left(\epsilon \delta_{\tau} \boldsymbol{E}_{h}^{k-\frac{1}{2}}, \boldsymbol{\phi}_{h}\right)-\left(\overline{\boldsymbol{H}}_{h}^{k-\frac{1}{2}}, \nabla \times \boldsymbol{\phi}_{h}\right)=0, & \forall \boldsymbol{\phi}_{h} \in \mathbf{V}_{h^{\prime}}^{0} \\
\left(\mu \delta_{\tau} \boldsymbol{H}_{h}^{k-\frac{1}{2}}, \boldsymbol{\psi}_{h}\right)+\left(\nabla \times \overline{\boldsymbol{E}}_{h}^{k-\frac{1}{2}}, \boldsymbol{\psi}_{h}\right)=0, & \forall \boldsymbol{\psi}_{h} \in \mathbf{U}_{h},
\end{array}
$$

subject to the initial conditions

$$
\boldsymbol{E}_{h}^{0}(\mathbf{x}, \mathbf{y})=\Pi_{h}^{c} \boldsymbol{E}_{0}(\mathbf{x}, \boldsymbol{y}), \quad \boldsymbol{H}_{h}^{0}(\mathbf{x}, \boldsymbol{y})=\Pi_{h}^{d} \boldsymbol{H}_{0}(\mathbf{x}, \mathbf{y}),
$$

where $\Pi_{h}^{d}$ denotes the $L^{2}$ projection into space $\mathbf{U}_{h}$ and $\Pi_{h}^{c}$ denotes the Nédélec interpolation on $\mathbf{V}_{h}^{0}$ (see [32,34]).

Note that the above scheme (2.3)-(2.4) can be written as follows:

$$
\begin{aligned}
& \left(\epsilon \boldsymbol{E}_{h}^{k}, \boldsymbol{\phi}_{h}\right)-\frac{\tau}{2}\left(\boldsymbol{H}_{h}^{k}, \nabla \times \boldsymbol{\phi}_{h}\right)=\left(\epsilon \boldsymbol{E}_{h}^{k-1}, \boldsymbol{\phi}_{h}\right)+\frac{\tau}{2}\left(\boldsymbol{H}_{h}^{k-1}, \nabla \times \boldsymbol{\phi}_{h}\right), \\
& \left(\mu \boldsymbol{H}_{h}^{k}, \boldsymbol{\psi}_{h}\right)+\frac{\tau}{2}\left(\nabla \times \boldsymbol{E}_{h}^{k}, \boldsymbol{\psi}_{h}\right)=\left(\mu \boldsymbol{H}_{h}^{k-1}, \boldsymbol{\psi}_{h}\right)-\frac{\tau}{2}\left(\nabla \times \boldsymbol{E}_{h}^{k-1}, \boldsymbol{\psi}_{h}\right) .
\end{aligned}
$$

Hence, at each time step, the coefficient matrix of (2.5)-(2.6) with the vector solution $\left(\boldsymbol{E}_{h}^{k}, \boldsymbol{H}_{h}^{k}\right)^{\top}$ can be written as $Q=\left(\begin{array}{cc}A & -B \\ B^{\top} & D\end{array}\right)$, which can be proved to be non-singular (cf. [32, Lemma 3.14]).

First, we have the following unconditional stability for our scheme.

Lemma 2.1. For the solution $\left(\boldsymbol{E}_{h}^{k}, \boldsymbol{H}_{h}^{k}\right)$ of (2.3)-(2.4) and any $k \in[1, K]$, we have

$$
\left\|\epsilon^{\frac{1}{2}} \boldsymbol{E}_{h}^{k}\right\|_{\boldsymbol{L}^{2}(D)}^{2}+\left\|\mu^{\frac{1}{2}} \boldsymbol{H}_{h}^{k}\right\|_{\boldsymbol{L}^{2}(D)}^{2}=\left\|\epsilon^{\frac{1}{2}} \boldsymbol{E}_{h}^{0}\right\|_{\boldsymbol{L}^{2}(D)}^{2}+\left\|\mu^{\frac{1}{2}} \boldsymbol{H}_{h}^{0}\right\|_{\boldsymbol{L}^{2}(D)}^{2} .
$$

Proof. Choosing $\boldsymbol{\phi}_{h}=\tau \overline{\boldsymbol{E}}_{h}^{k-\frac{1}{2}}$ and $\boldsymbol{\psi}_{h}=\tau \overline{\boldsymbol{H}}_{h}^{k-\frac{1}{2}}$ in (2.3) and (2.4), respectively, and adding the results together, we have

$$
\frac{1}{2}\left(\left\|\epsilon^{\frac{1}{2}} \boldsymbol{E}_{h}^{k}\right\|_{\boldsymbol{L}^{2}(D)}^{2}-\left\|\epsilon^{\frac{1}{2}} \boldsymbol{E}_{h}^{k-1}\right\|_{\boldsymbol{L}^{2}(D)}^{2}\right)+\frac{1}{2}\left(\left\|\mu^{\frac{1}{2}} \boldsymbol{H}_{h}^{k}\right\|_{\boldsymbol{L}^{2}(D)}^{2}-\left\|\mu^{\frac{1}{2}} \boldsymbol{H}_{h}^{k-1}\right\|_{\boldsymbol{L}^{2}(D)}^{2}\right)=0,
$$

which concludes the proof.

Denote $C_{v}=\frac{1}{\sqrt{\epsilon \mu}}$ for the wave propagation speed in a medium with permittivity $\epsilon$ and permeability $\mu$. Then we can prove the following optimal error estimate for our scheme. 
Theorem 2.1. Suppose that the solution $(\boldsymbol{E}, \boldsymbol{H})$ of (2.1)-(2.2) satisfy the following regularity:

$$
\begin{aligned}
& \epsilon^{\frac{1}{2}} \boldsymbol{E} \in \boldsymbol{L}^{\infty}\left(0, T ; \boldsymbol{H}^{r}(\text { curl } ; D)\right), \epsilon^{\frac{1}{2}} \partial_{t} \boldsymbol{E} \in \boldsymbol{L}^{2}\left(0, T ; \boldsymbol{H}^{r}(\text { curl } ; D)\right), \epsilon^{\frac{1}{2}} \nabla \times \partial_{t}^{2} \boldsymbol{E} \in \boldsymbol{L}^{2}\left(0, T ; \boldsymbol{L}^{2}(D)\right), \\
& \boldsymbol{\mu}^{\frac{1}{2}} \boldsymbol{H} \in \boldsymbol{L}^{\infty}\left(0, T ; \boldsymbol{H}^{r}(D)\right), \mu^{\frac{1}{2}} \nabla \times \partial_{t}^{2} \boldsymbol{H} \in \boldsymbol{L}^{2}\left(0, T ; \boldsymbol{L}^{2}(D)\right),
\end{aligned}
$$

then for any $k \in[1, K]$, we have

$$
\begin{aligned}
& \left\|\epsilon^{\frac{1}{2}}\left(\boldsymbol{E}_{h}^{k}-\boldsymbol{E}\left(\boldsymbol{x}, t_{k} ; \boldsymbol{y}\right)\right)\right\|_{\boldsymbol{L}^{2}(D)}+\left\|\mu^{\frac{1}{2}}\left(\boldsymbol{H}_{h}^{k}-\boldsymbol{H}\left(\boldsymbol{x}, \boldsymbol{t}_{k} ; \boldsymbol{y}\right)\right)\right\|_{\boldsymbol{L}^{2}(D)} \\
\leq & C h^{r}\left(\left\|\epsilon^{1 / 2} \partial_{t} \boldsymbol{E}\right\|_{\left.\boldsymbol{L}_{(0, T}^{2} \boldsymbol{H}^{r}(\mathrm{curl} ; D)\right)}+\left\|\epsilon^{1 / 2} \boldsymbol{E}\right\|_{\left.\boldsymbol{L}_{(0, T}^{\infty} ; \boldsymbol{H}^{r}(\mathrm{curl} ; D)\right)}+\left\|\mu^{1 / 2} \boldsymbol{H}\right\|_{\left.\boldsymbol{L}_{(0, T ;}^{\infty} \boldsymbol{H}^{r}(D)\right)}\right) \\
& +C \tau^{2}\left(\left\|\mu^{1 / 2} \nabla \times\left(\partial_{t}^{2} \boldsymbol{H}\right)\right\|_{\left.\boldsymbol{L}_{(0, T ;}^{2} \boldsymbol{L}^{2}(D)\right)}+\left\|\epsilon^{1 / 2} \nabla \times\left(\partial_{t}^{2} \boldsymbol{E}\right)\right\|_{\left.\boldsymbol{L}_{(0, T ;}^{2} \boldsymbol{L}^{2}(D)\right)}\right),
\end{aligned}
$$

where the constant $C>0$ is independent of $h$ and $\tau$, and $r \geq 1$ is the degree of the finite element spaces $\mathbf{V}_{h}^{0}$ and $\mathbf{U}_{h}$.

Proof. Integrating (1.1) and (1.2) from $t_{k-1}$ to $t_{k}$, multiplying the results by $\boldsymbol{\phi}_{h} \in \mathbf{V}_{h}^{0}$ and $\psi_{h} \in \mathbf{U}_{h}$ and integrating over $D$, respectively, we obtain

$$
\begin{aligned}
& \left(\epsilon \frac{\boldsymbol{E}^{k}-\boldsymbol{E}^{k-1}}{\tau}, \boldsymbol{\phi}_{h}\right)-\left(\frac{1}{\tau} \int_{I_{k}} \boldsymbol{H} d s, \nabla \times \boldsymbol{\phi}_{h}\right)=0, \\
& \left(\mu \frac{\boldsymbol{H}^{k}-\boldsymbol{H}^{k-1}}{\tau}, \boldsymbol{\psi}_{h}\right)+\left(\frac{1}{\tau} \int_{I_{k}} \nabla \times \boldsymbol{E} d s, \boldsymbol{\psi}_{h}\right)=0,
\end{aligned}
$$

where for simplicity we denote $u^{j}=u\left(t_{j}\right)$ for $u=E$ and $\boldsymbol{H}$.

Let us introduce the errors

$$
\begin{aligned}
& \widehat{\boldsymbol{E}}_{h}^{k}:=\boldsymbol{E}_{h}^{k}-\boldsymbol{E}^{k}=\left(\boldsymbol{E}_{h}^{k}-\Pi_{h}^{c} \boldsymbol{E}^{k}\right)-\left(\boldsymbol{E}^{k}-\Pi_{h}^{c} \boldsymbol{E}^{k}\right)=\boldsymbol{E}_{h \xi}^{k}-\boldsymbol{E}_{h \eta^{\prime}}^{k} \\
& \widehat{\boldsymbol{H}}_{h}^{k}:=\boldsymbol{H}_{h}^{k}-\boldsymbol{H}^{k}=\left(\boldsymbol{H}_{h}^{k}-\Pi_{h}^{d} \boldsymbol{H}^{k}\right)-\left(\boldsymbol{H}^{k}-\Pi_{h}^{d} \boldsymbol{H}^{k}\right)=\boldsymbol{H}_{h \xi}^{k}-\boldsymbol{H}_{h \eta}^{k} .
\end{aligned}
$$

Subtracting (2.8)-(2.9) from (2.5)-(2.6), respectively, we obtain the error equations:

$$
\begin{aligned}
& \left(\epsilon \frac{\widehat{\boldsymbol{E}}_{h}^{k}-\widehat{\boldsymbol{E}}_{h}^{k-1}}{\tau}, \boldsymbol{\phi}_{h}\right)-\left(\frac{\widehat{\boldsymbol{H}}_{h}^{k}+\widehat{\boldsymbol{H}}_{h}^{k-1}}{2}, \nabla \times \boldsymbol{\phi}_{h}\right)=\left(\frac{\boldsymbol{H}^{k}+\boldsymbol{H}^{k-1}}{2}-\frac{1}{\tau} \int_{I_{k}} \boldsymbol{H} d s, \nabla \times \boldsymbol{\phi}_{h}\right), \\
& \left(\mu \frac{\widehat{\boldsymbol{H}}_{h}^{k}-\widehat{\boldsymbol{H}}_{h}^{k-1}}{\tau}, \boldsymbol{\psi}_{h}\right)+\left(\nabla \times \frac{\widehat{\boldsymbol{E}}_{h}^{k}+\widehat{\boldsymbol{E}}_{h}^{k-1}}{2}, \boldsymbol{\psi}_{h}\right)=\left(\frac{1}{\tau} \int_{I_{k}} \nabla \times \boldsymbol{E} d s-\nabla \times \frac{\boldsymbol{E}^{k}+\boldsymbol{E}^{k-1}}{2}, \boldsymbol{\psi}_{h}\right) .
\end{aligned}
$$

Using the error decomposition (2.10)-(2.11), we can rewrite the above error equations as follows:

$$
\begin{aligned}
& \left(\epsilon \delta_{\tau} \boldsymbol{E}_{h \xi}^{k-\frac{1}{2}}, \boldsymbol{\phi}_{h}\right)-\left(\overline{\boldsymbol{H}}_{h \xi}^{k-\frac{1}{2}}, \nabla \times \boldsymbol{\phi}_{h}\right) \\
= & \left(\epsilon \delta_{\tau} \boldsymbol{E}_{h \eta}^{k-\frac{1}{2}}, \boldsymbol{\phi}_{h}\right)-\left(\overline{\boldsymbol{H}}_{h \eta}^{k-\frac{1}{2}}, \nabla \times \boldsymbol{\phi}_{h}\right)+\left(\overline{\boldsymbol{H}}^{k-\frac{1}{2}}-\frac{1}{\tau} \int_{I_{k}} \boldsymbol{H} d s, \nabla \times \boldsymbol{\phi}_{h}\right), \\
& \left(\mu \delta_{\tau} \boldsymbol{H}_{h \xi}^{k-\frac{1}{2}}, \boldsymbol{\psi}_{h}\right)+\left(\nabla \times \overline{\boldsymbol{E}}_{h \xi}^{k-\frac{1}{2}}, \boldsymbol{\psi}_{h}\right) \\
= & \left(\mu \delta_{\tau} \boldsymbol{H}_{h \eta}^{k-\frac{1}{2}}, \boldsymbol{\psi}_{h}\right)+\left(\nabla \times \overline{\boldsymbol{E}}_{h \eta}^{k-\frac{1}{2}}, \boldsymbol{\psi}_{h}\right)+\left(\frac{1}{\tau} \int_{I_{k}} \nabla \times \boldsymbol{E} d s-\nabla \times \overline{\boldsymbol{E}}^{k-\frac{1}{2}}, \boldsymbol{\psi}_{h}\right) .
\end{aligned}
$$


Choosing $\boldsymbol{\phi}_{h}=2 \tau \overline{\boldsymbol{E}}_{h \xi^{\tau}}^{k-\frac{1}{2}}$ in (2.14) and $\boldsymbol{\psi}_{h}=2 \tau \overline{\boldsymbol{H}}_{h \xi^{2}}^{k-\frac{1}{2}}$ in (2.15), then adding the resultants, we obtain

$$
\begin{aligned}
& \left(\left\|\epsilon^{1 / 2} \boldsymbol{E}_{h \xi}^{k}\right\|^{2}-\left\|\epsilon^{1 / 2} \boldsymbol{E}_{h \xi}^{k-1}\right\|^{2}\right)+\left(\left\|\mu^{1 / 2} \boldsymbol{H}_{h \xi}^{k}\right\|^{2}-\left\|\mu^{1 / 2} \boldsymbol{H}_{h \xi}^{k-1}\right\|^{2}\right) \\
= & 2 \tau\left(\epsilon \delta_{\tau} \boldsymbol{E}_{h \eta}^{k-\frac{1}{2}}, \overline{\boldsymbol{E}}_{h \xi}^{k-\frac{1}{2}}\right)-2 \tau\left(\overline{\boldsymbol{H}}_{h \eta}^{k-\frac{1}{2}}, \nabla \times \overline{\boldsymbol{E}}_{h \xi}^{k-\frac{1}{2}}\right)+2 \tau\left(\overline{\boldsymbol{H}}^{k-\frac{1}{2}}-\frac{1}{\tau} \int_{I_{k}} \boldsymbol{H} d s, \nabla \times \overline{\boldsymbol{E}}_{h \xi}^{k-\frac{1}{2}}\right) \\
& +2 \tau\left(\mu \delta_{\tau} \boldsymbol{H}_{h \eta}^{k-\frac{1}{2}}, \overline{\boldsymbol{H}}_{h \xi}^{k-\frac{1}{2}}\right)+2 \tau\left(\nabla \times \overline{\boldsymbol{E}}_{h \eta}^{k-\frac{1}{2}}, \overline{\boldsymbol{H}}_{h \xi}^{k-\frac{1}{2}}\right)+2 \tau\left(\frac{1}{\tau} \int_{I_{k}} \nabla \times \boldsymbol{E} d s-\nabla \times \overline{\boldsymbol{E}}^{k-\frac{1}{2}}, \overline{\boldsymbol{H}}_{h \xi}^{k-\frac{1}{2}}\right) \\
:= & \sum_{i=1}^{6} \operatorname{Err}_{i} .
\end{aligned}
$$

By the Cauchy-Schwarz inequality, the following estimate [32, Lemma 3.16]:

$$
\begin{aligned}
\left\|\delta_{\tau} u^{k-\frac{1}{2}}\right\|_{L_{2}(D)}^{2} & :=\left\|\frac{u^{k}-u^{k-1}}{\tau}\right\|_{L_{2}(D)}^{2} \\
& \leq \frac{1}{\tau} \int_{t_{k-1}}^{t_{k}}\left\|\partial_{t} u\right\|_{L_{2}(D)}^{2} d s, \quad \forall u \in H^{1}\left(\left(t_{k-1}, t_{k}\right) ; L^{2}(D)\right),
\end{aligned}
$$

and the interpolation error estimates: for any $r \geq 1$, any $\boldsymbol{u} \in \boldsymbol{H}^{r}(\mathrm{curl} ; D)$,

$$
\begin{aligned}
& \left\|\boldsymbol{u}-\Pi_{h}^{c} \boldsymbol{u}\right\|_{\boldsymbol{L}_{2}(D)}+\left\|\nabla \times\left(\boldsymbol{u}-\Pi_{h}^{c} \boldsymbol{u}\right)\right\|_{\boldsymbol{L}_{2}(D)} \leq C h^{r}\|\boldsymbol{u}\|_{\boldsymbol{H}^{r}(\mathrm{curl} ; D)^{\prime}} \\
& \left\|\boldsymbol{v}-\Pi_{h}^{d} \boldsymbol{v}\right\|_{\boldsymbol{L}_{2}(D)} \leq C h^{r}\|\boldsymbol{u}\|_{\boldsymbol{H}^{r}(D)^{\prime}}, \quad \forall \boldsymbol{v} \in \boldsymbol{H}^{r}(D),
\end{aligned}
$$

we have

$$
\begin{aligned}
E r r_{1} & \leq 2 \tau\left\|\epsilon^{1 / 2} \delta_{\tau} \boldsymbol{E}_{h \eta}^{k-\frac{1}{2}}\right\|_{\boldsymbol{L}_{2}(D)}\left\|\epsilon^{1 / 2} \overline{\boldsymbol{E}}_{h \xi^{\frac{2}{2}}}^{k-\frac{1}{2}}\right\|_{\boldsymbol{L}_{2}(D)} \\
& \leq \tau\left(\frac{1}{4 \delta_{*}}\left\|\epsilon^{1 / 2} \delta_{\tau} \boldsymbol{E}_{h \eta}^{k-\frac{1}{2}}\right\|_{\boldsymbol{L}_{2}(D)}^{2}+4 \delta_{*}\left\|\epsilon^{1 / 2} \overline{\boldsymbol{E}}_{h \xi^{2}}^{k-\frac{1}{2}}\right\|_{\boldsymbol{L}_{2}(D)}^{2}\right) \\
& \leq \frac{1}{4 \delta_{*}} \int_{I_{k}}\left\|\epsilon^{1 / 2} \partial_{t} \boldsymbol{E}_{h \eta}\right\|_{\boldsymbol{L}_{2}(D)}^{2} d s+2 \tau \delta_{*}\left(\left\|\epsilon^{1 / 2} \boldsymbol{E}_{h \xi}^{k}\right\|_{\boldsymbol{L}_{2}(D)}^{2}+\left\|\epsilon^{1 / 2} \boldsymbol{E}_{h \xi^{\tau}}^{k-1}\right\|_{\boldsymbol{L}_{2}(D)}^{2}\right) \\
& \leq \frac{1}{\delta_{*}} \int_{I_{k}} C h^{2 r}\left\|\epsilon^{1 / 2} \partial_{t} \boldsymbol{E}\right\|_{\boldsymbol{H}^{r}(\mathrm{curl} ; D)}^{2} d s+4 \tau \delta_{*} \max _{0 \leq k \leq K}\left\|\epsilon^{1 / 2} \boldsymbol{E}_{h \xi}^{k}\right\|_{\boldsymbol{L}_{2}(D)^{\prime}}^{2}
\end{aligned}
$$

where we used the arithmetic-geometric inequality in the second inequality.

By the definition of projection $\Pi_{h}^{d}$ and the property $\nabla \times \overline{\boldsymbol{E}}_{h \xi}^{k-\frac{1}{2}} \subseteq \boldsymbol{U}_{h}$, we have

$$
E r r_{2}=0, \quad E r r_{4}=0 .
$$

Using integration by parts, the following estimate [32, Lemma 3.16]:

$$
\begin{aligned}
& \left\|\frac{u\left(t_{k-1}\right)+u\left(t_{k}\right)}{2}-\frac{1}{\tau} \int_{I_{k}} u(s) d s\right\|_{L^{2}(D)} \\
\leq & \frac{\tau^{3}}{4} \int_{t_{k-1}}^{t_{k}}\left\|\partial_{t}^{2} u\right\|_{L_{2}(D)}^{2} d s, \quad \forall u \in H^{2}\left(\left(t_{k-1}, t_{k}\right) ; L^{2}(D)\right),
\end{aligned}
$$


and the Cauchy-Schwarz inequality, we have

$$
\begin{aligned}
E r r_{3} & \leq 2 \tau\left(\nabla \times\left(\overline{\boldsymbol{H}}^{k-\frac{1}{2}}-\frac{1}{\tau} \int_{I_{k}} \boldsymbol{H} d s\right), \overline{\boldsymbol{E}}_{h \tau^{2}}^{k-\frac{1}{2}}\right) \\
& \leq 2 \tau C_{v}\left(\frac{1}{8 \delta_{*}}\left\|\mu^{1 / 2}\left(\overline{\nabla \times \boldsymbol{H}^{k-\frac{1}{2}}}-\frac{1}{\tau} \int_{I_{k}} \nabla \times \boldsymbol{H} d s\right)\right\|_{\boldsymbol{L}_{2}(D)}^{2}+2 \delta_{*}\left\|\epsilon^{1 / 2} \overline{\boldsymbol{E}}_{h \xi^{2}}^{k-\frac{1}{2}}\right\|_{\boldsymbol{L}_{2}(D)}^{2}\right) \\
& \leq 2 \tau C_{v}\left(\frac{\tau^{3}}{32 \delta_{*}} \int_{I_{k}}\left\|\mu^{1 / 2} \nabla \times\left(\partial_{t}^{2} \boldsymbol{H}\right)\right\|_{\boldsymbol{L}_{2}(D)}^{2} d s+\delta_{*}\left(\left\|\epsilon^{1 / 2} \boldsymbol{E}_{h \xi}^{k}\right\|_{\boldsymbol{L}_{2}(D)}^{2}+\left\|\epsilon^{1 / 2} \boldsymbol{E}_{h \xi}^{k-1}\right\|_{\boldsymbol{L}_{2}(D)}^{2}\right)\right) \\
& \leq \frac{\tau^{4} C_{v}}{16 \delta_{*}} \int_{I_{k}}\left\|\mu^{1 / 2} \nabla \times\left(\partial_{t}^{2} \boldsymbol{H}\right)\right\|_{\boldsymbol{L}_{2}(D)}^{2} d s+4 \tau \delta_{*} C_{v} \max _{0 \leq k \leq K}\left\|\epsilon^{1 / 2} \boldsymbol{E}_{h \xi}^{k}\right\|_{\boldsymbol{L}_{2}(D)}^{2} .
\end{aligned}
$$

Using the Cauchy-Schwarz inequality and the interpolation error estimate (2.18), we have

$$
\begin{aligned}
E r r_{5} & \leq 2 \tau\left\|\nabla \times \overline{\boldsymbol{E}}_{h \eta}^{k-\frac{1}{2}}\right\|_{\boldsymbol{L}_{2}(D)}\left\|\overline{\boldsymbol{H}}_{h \zeta}^{k-\frac{1}{2}}\right\|_{\boldsymbol{L}_{2}(D)} \\
& \leq 2 \tau C_{v}\left(\frac{1}{8 \delta_{*}}\left\|\epsilon^{1 / 2} \nabla \times \overline{\boldsymbol{E}}_{h \eta}^{k-\frac{1}{2}}\right\|_{\boldsymbol{L}_{2}(D)}^{2}+2 \delta_{*}\left\|\mu^{1 / 2} \overline{\boldsymbol{H}}_{h \xi}^{k-\frac{1}{2}}\right\|_{\boldsymbol{L}_{2}(D)}^{2}\right) \\
& \leq \frac{\tau C_{v} C h^{2 r}}{\delta_{*}}\left\|\epsilon^{1 / 2} \boldsymbol{E}\right\|_{\left.\boldsymbol{L}_{(0, T ;}^{\infty} \boldsymbol{H}^{r}(\mathrm{curl} ; D)\right)}^{2}+4 \tau C_{v} \delta_{*} \max _{0 \leq k \leq K}\left\|\mu^{1 / 2} \boldsymbol{H}_{h \xi}^{k}\right\|_{\boldsymbol{L}_{2}(D)}^{2} .
\end{aligned}
$$

By the Cauchy-Schwarz inequality and (2.20), we have

$$
\begin{aligned}
E r r_{6} & \leq 2 \tau C_{v}\left(\frac{1}{8 \delta_{*}}\left\|\epsilon^{1 / 2}\left(\frac{1}{\tau} \int_{I_{k}} \nabla \times \boldsymbol{E} d s-\overline{\nabla \times \boldsymbol{E}^{k-\frac{1}{2}}}\right)\right\|_{\boldsymbol{L}_{2}(D)}^{2}+2 \delta_{*}\left\|\mu^{1 / 2} \overline{\boldsymbol{H}}_{h \xi}^{k-\frac{1}{2}}\right\|_{\boldsymbol{L}_{2}(D)}^{2}\right) \\
& \leq \frac{\tau^{4} C_{v}}{16 \delta_{*}} \int_{I_{k}}\left\|\epsilon^{1 / 2} \nabla \times\left(\partial_{t}^{2} \boldsymbol{E}\right)\right\|_{\boldsymbol{L}_{2}(D)}^{2} d s+4 \tau C_{v} \delta_{*} \max _{0 \leq k \leq K}\left\|\mu^{1 / 2} \boldsymbol{H}_{h \xi}^{k}\right\|_{\boldsymbol{L}_{2}(D)}^{2} .
\end{aligned}
$$

Substituting the above estimates of $E r r_{i}$ into (2.16), then summing up the resultant from $k=1$ to $n \leq K$ and using the fact that $n \tau \leq T$, we have

$$
\begin{aligned}
& \left(\left\|\epsilon^{\frac{1}{2}} \boldsymbol{E}_{h \xi}^{n}\right\|_{\boldsymbol{L}_{2}(D)}^{2}-\left\|\epsilon^{\frac{1}{2}} \boldsymbol{E}_{h \xi}^{0}\right\|_{\boldsymbol{L}_{2}(D)}^{2}\right)+\left(\left\|\mu^{\frac{1}{2}} \boldsymbol{H}_{h \xi}^{n}\right\|_{\boldsymbol{L}_{2}(D)}^{2}-\left\|\mu^{\frac{1}{2}} \boldsymbol{H}_{h \xi}^{0}\right\|_{\boldsymbol{L}_{2}(D)}^{2}\right) \\
\leq & \frac{C h^{2 r}}{\delta_{*}} \int_{0}^{t_{n}}\left\|\epsilon^{1 / 2} \partial_{t} \boldsymbol{E}\right\|_{\boldsymbol{H}^{r}(\mathrm{curl} ; D)}^{2} d s+4 T \delta_{*}\left\|\epsilon^{1 / 2} \boldsymbol{E}_{h \xi}\right\|_{\infty}^{2} \\
& +\frac{\tau^{4} C_{v}}{16 \delta_{*}} \int_{0}^{t_{n}}\left\|\mu^{1 / 2} \nabla \times\left(\partial_{t}^{2} \boldsymbol{H}\right)\right\|_{\boldsymbol{L}_{2}(D)}^{2} d s+4 T \delta_{*} C_{v}\left\|\epsilon^{1 / 2} \boldsymbol{E}_{h \xi}\right\|_{\infty}^{2} \\
& +\frac{T C_{v} C h^{2 r}}{\delta_{*}}\left\|\epsilon^{1 / 2} \boldsymbol{E}\right\|_{\boldsymbol{L}_{(0, T ;} \boldsymbol{H}_{(\mathrm{curl} ; D)}}^{2}+4 T C_{v} \delta_{*}\left\|\mu^{1 / 2} \boldsymbol{H}_{h \xi}\right\|_{\infty}^{2} \\
& +\frac{\tau^{4} C_{v}}{16 \delta_{*}} \int_{0}^{t_{n}}\left\|\epsilon^{1 / 2} \nabla \times\left(\partial_{t}^{2} \boldsymbol{E}\right)\right\|_{\boldsymbol{L}_{2}(D)}^{2} d s+4 T C_{v} \delta_{*}\left\|\mu^{1 / 2} \boldsymbol{H}_{h \xi}\right\|_{\infty}^{2},
\end{aligned}
$$

where we denote

$$
\left\|\epsilon^{1 / 2} \boldsymbol{E}_{h \xi}\right\|_{\infty}:=\max _{0 \leq k \leq K}\left\|\epsilon^{1 / 2} \boldsymbol{E}_{h \xi}^{k}\right\|, \quad\left\|\mu^{1 / 2} \boldsymbol{H}_{h \xi}\right\|_{\infty}:=\max _{0 \leq k \leq K}\left\|\mu^{1 / 2} \boldsymbol{H}_{h \xi}^{k}\right\|_{\boldsymbol{L}_{2}(D)} .
$$


Taking the maximum of (2.21) with respect to $n$, then choosing $\delta_{*}$ small enough (e.g., $\left.4 T \delta_{*} \max \left(1, C_{v}\right) \leq \frac{1}{3}\right)$, we have

$$
\begin{aligned}
& \left\|\epsilon^{\frac{1}{2}} \boldsymbol{E}_{h \xi}\right\|_{\infty}+\left\|\mu^{\frac{1}{2}} \boldsymbol{H}_{h \xi}\right\|_{\infty} \\
\leq & C h^{r}\left(\left\|\epsilon^{1 / 2} \partial_{t} \boldsymbol{E}\right\|_{\boldsymbol{L}^{2}\left(0, T ; \boldsymbol{H}^{r}(\text { curl; } ;)\right)}+\left\|\epsilon^{1 / 2} \boldsymbol{E}\right\|_{\boldsymbol{L}^{\infty}\left(0, T ; \boldsymbol{H}^{r}(\mathrm{curl} ; D)\right)}\right) \\
& +C \tau^{2}\left(\left\|\mu^{1 / 2} \nabla \times\left(\partial_{t}^{2} \boldsymbol{H}\right)\right\|_{\left.\boldsymbol{L}_{(0, T ;}^{2} \boldsymbol{L}^{2}(D)\right)}+\left\|\epsilon^{1 / 2} \nabla \times\left(\partial_{t}^{2} \boldsymbol{E}\right)\right\|_{\boldsymbol{L}^{2}\left(0, T ; \boldsymbol{L}^{2}(D)\right)}\right) .
\end{aligned}
$$

Using the interpolation error estimates (2.18)-(2.19), we obtain

$$
\begin{aligned}
& \left\|\epsilon^{\frac{1}{2}} \boldsymbol{E}_{h \eta}\right\|_{\boldsymbol{L}^{2}(D)}+\left\|\mu^{\frac{1}{2}} \boldsymbol{H}_{h \eta}\right\|_{\boldsymbol{L}^{2}(D)} \\
\leq & C h^{r}\left(\left\|\epsilon^{1 / 2} \boldsymbol{E}\right\|_{\left.\boldsymbol{L}_{(0, T ;}^{\infty} \boldsymbol{H}^{r}(\text { curl } ; D)\right)}+\left\|\mu^{1 / 2} \boldsymbol{H}\right\|_{\left.\boldsymbol{L}_{\left(0, T ; \boldsymbol{H}^{r}(\text { curl } ; D)\right)}\right) .}\right.
\end{aligned}
$$

Combining the estimates of (2.22) and (2.23), and using the triangle inequality, we complete the proof.

\subsection{Analysis of single level Monte Carlo method}

In practice, we are often interested in estimating the expected value (also known as expectation) of the random solutions. The expectation $\mathbb{E}[\mathbf{u}]$ can be estimated by a sample mean over the solution samples $\left\{\widehat{\mathbf{u}}^{i}\right\}, i=1,2, \cdots M$, corresponding to $M$ i.i.d. realizations of the random inputs:

$$
\mathbb{E}[\mathbf{u}] \approx E_{M}[\mathbf{u}]:=\frac{1}{M} \sum_{i=1}^{M} \widehat{\mathbf{u}}^{i} .
$$

Here $\mathbf{u}$ can denote either the analytic solutions $\boldsymbol{E}$ and $\boldsymbol{H}$, or the finite element solutions $\boldsymbol{E}_{h}$ and $\boldsymbol{H}_{h}$.

The following result was proved in [4] and gives a bound on the statistical error for the Monte Carlo estimator (2.24).

Lemma 2.2. [4, Lemma 4.1] For any $M \in \mathbb{N}$ and $\mathbf{u} \in L^{2}\left(U ; L^{2}(D)\right)$, we have

$$
\left\|\mathbb{E}[\mathbf{u}]-E_{M}[\mathbf{u}]\right\|_{L^{2}(D \times U)} \leq \frac{1}{\sqrt{M}}\|\mathbf{u}\|_{L^{2}(D \times U)} .
$$

Remark 2.1. If we define the variance of a function $\mathbf{u}$ as

$$
\sigma(\mathbf{u}):=\sqrt{\mathbb{E}\left[\|\mathbf{u}\|_{L^{2}(D)}^{2}\right]-\|\mathbb{E}[\mathbf{u}]\|_{L^{2}(D)^{\prime}}^{\prime}}
$$

then we have a more accurate statistical error estimate for the Monte Carlo method (cf. the proof of [4, Lemma 4.1]):

$$
\left\|\mathbb{E}[\mathbf{u}]-E_{M}[\mathbf{u}]\right\|_{L^{2}(D \times U)}=\frac{1}{\sqrt{M}} \sigma(\mathbf{u}) .
$$


The single level Monte Carlo method is to find out the estimator $E_{M}[\mathbf{u}]$ defined in (2.24). To this end, we pick a sequence of i.i.d. sample points $y^{i}, i=1,2, \cdots M$, and compute the corresponding numerical solution $\widehat{\mathbf{u}}^{i}$ of (2.5)-(2.6). The error estimate of single level Monte Carlo finite element method is given by the following theorem:

Theorem 2.2. Under the same regularity assumptions given in Theorem 2.1, the single level Monte Carlo method (2.5)-(2.6) satisfies the following error estimate: at any time step $t_{k}=k \tau$, $k=1,2, \cdots, K$, we have

$$
\left\|\mathbb{E}\left[\boldsymbol{E}\left(t_{k}\right)\right]-E_{M}\left[\boldsymbol{E}_{h}^{k}\right]\right\|_{L^{2}(D \times U)}+\left\|\mathbb{E}\left[\boldsymbol{H}\left(t_{k}\right)\right]-E_{M}\left[\boldsymbol{H}_{h}^{k}\right]\right\|_{L^{2}(D \times U)} \leq C\left(\tau^{2}+h^{r}+M^{-1 / 2}\right) .
$$

Proof. For simplicity, we denote $\boldsymbol{E}^{k}:=\boldsymbol{E}\left(\boldsymbol{x}, \boldsymbol{t}_{k}\right)$ and $\boldsymbol{H}^{k}:=\boldsymbol{H}\left(\boldsymbol{x}, \boldsymbol{t}_{k}\right)$.

Using Jensen's inequality for the solution $E$, we have

$$
\begin{aligned}
\left\|\mathbb{E}\left[\boldsymbol{E}\left(t_{k}\right)\right]-E_{M}\left[\boldsymbol{E}_{h}^{k}\right]\right\|_{L^{2}(D \times U)} & \leq\left\|\mathbb{E}\left[\boldsymbol{E}\left(t_{k}\right)\right]-\mathbb{E}\left[\boldsymbol{E}_{h}^{k}\right]\right\|_{L^{2}(D \times U)}+\left\|\mathbb{E}\left[\boldsymbol{E}_{h}^{k}\right]-E_{M}\left[\boldsymbol{E}_{h}^{k}\right]\right\|_{L^{2}(D \times U)} \\
& \leq \mathbb{E}\left[\left\|\boldsymbol{E}\left(t_{k}\right)-\boldsymbol{E}_{h}^{k}\right\|_{\boldsymbol{L}^{2}(D)}^{2}\right]^{1 / 2}+\left\|\mathbb{E}\left[\boldsymbol{E}_{h}^{k}\right]-E_{M}\left[\boldsymbol{E}_{h}^{k}\right]\right\|_{L^{2}(D \times U)}
\end{aligned}
$$

which, along with a similar estimate for the solution $\boldsymbol{H}$, leads to

$$
\left\|\mathbb{E}\left[\boldsymbol{E}\left(t_{k}\right)\right]-E_{M}\left[\boldsymbol{E}_{h}^{k}\right]\right\|_{L^{2}(D \times U)}+\left\|\mathbb{E}\left[\boldsymbol{H}\left(t_{k}\right)\right]-E_{M}\left[\boldsymbol{H}_{h}^{k}\right]\right\|_{L^{2}(D \times U)} \leq \sqrt{2} I+I I,
$$

where we denote

$$
\begin{aligned}
& I:=\mathbb{E}\left[\left\|\boldsymbol{E}\left(t_{k}\right)-\boldsymbol{E}_{h}^{k}\right\|_{\boldsymbol{L}^{2}(D)}^{2}+\left\|\boldsymbol{H}\left(t_{k}\right)-\boldsymbol{H}_{h}^{k}\right\|_{\boldsymbol{L}^{2}(D)}^{2}\right]^{1 / 2}, \\
& I I:=\left\|\mathbb{E}\left[\boldsymbol{E}_{h}^{k}\right]-E_{M}\left[\boldsymbol{E}_{h}^{k}\right]\right\|_{L^{2}(D \times U)}+\left\|\mathbb{E}\left[\boldsymbol{H}_{h}^{k}\right]-E_{M}\left[\boldsymbol{H}_{h}^{k}\right]\right\|_{L^{2}(D \times U)} .
\end{aligned}
$$

The first term $I$ in (2.25) measures the error of the finite element scheme and the second term II gives the statistical error. Note that the estimate of $I$ is given by Theorem 2.1, with the constant coefficient $C$ independent of vector $y$ after taking the mean.

To bound the term $I I$, we use Lemma 2.2 and Lemma 2.1 to obtain

$$
\begin{aligned}
& \epsilon^{-}\left\|\mathbb{E}\left[\boldsymbol{E}_{h}^{k}\right]-E_{M}\left[\boldsymbol{E}_{h}^{k}\right]\right\|_{L^{2}(D \times U)}^{2}+\mu^{-}\left\|\mathbb{E}\left[\boldsymbol{H}_{h}^{k}\right]-E_{M}\left[\boldsymbol{H}_{h}^{k}\right]\right\|_{L^{2}(D \times U)}^{2} \\
\leq & \frac{1}{M}\left(\int_{D} \int_{U} \epsilon\left|\boldsymbol{E}_{h}^{k}\right|^{2}+\mu\left|\boldsymbol{H}_{h}^{k}\right|^{2} d \boldsymbol{y} d \boldsymbol{x}\right) \\
\leq & \frac{1}{M}\left(\int_{D} \int_{U} \epsilon\left|\boldsymbol{E}_{h}^{0}\right|^{2}+\mu\left|\boldsymbol{H}_{h}^{0}\right|^{2} d \boldsymbol{y} d \boldsymbol{x}\right) \leq \frac{1}{M}\left(\epsilon^{+}\left\|\boldsymbol{E}_{h}^{0}\right\|_{L^{2}(D \times U)}^{2}+\mu^{+}\left\|\boldsymbol{H}_{h}^{0}\right\|_{L^{2}(D \times U)}^{2}\right) \leq \frac{C}{M} .
\end{aligned}
$$

This leads to $I I \leq C M^{-1 / 2}$. Substituting the estimates of $I$ and $I I$ into (2.25) concludes our proof. 


\subsection{Analysis of multi-level Monte Carlo method}

For the multi-level Monte Carlo (MLMC) method we discretize the physical domain $D$ by a sequence of nested partitions $\left\{\mathcal{T}_{l}\right\}_{l=1}^{L}$ with corresponding mesh size $h_{l}$ and time step $\tau_{l}$, and then solve the finite element scheme (2.5)-(2.6) in the corresponding mixed finite element spaces $\mathbf{U}_{h_{l}}$ and $\mathbf{V}_{h_{l}}$. Introducing the notation $\mathbf{u}_{0}:=0$, we can write

$$
\mathbf{u}_{L}=\sum_{l=1}^{L}\left(\mathbf{u}_{l}-\mathbf{u}_{l-1}\right)
$$

where $\mathbf{u}_{l}$ represents the solution obtained on mesh $\mathcal{T}_{l}$.

By the linearity of expectation, we have

$$
\mathbb{E}\left[\mathbf{u}_{L}\right]=\mathbb{E}\left[\sum_{l=1}^{L}\left(\mathbf{u}_{l}-\mathbf{u}_{l-1}\right)\right]=\sum_{l=1}^{L} \mathbb{E}\left[\mathbf{u}_{l}-\mathbf{u}_{l-1}\right] .
$$

In the MLMC method, we estimate $\mathbb{E}\left[\mathbf{u}_{l}-\mathbf{u}_{l-1}\right]$ by a level dependent number of samples $M_{l}$, i.e, the MLMC estimator is given by:

$$
\mathbb{E}\left[\mathbf{u}_{L}\right] \approx E^{M L}[\mathbf{u}]:=\sum_{l=1}^{L} E_{M_{l}}\left[\mathbf{u}_{l}-\mathbf{u}_{l-1}\right]
$$

Theorem 2.3. Under the same assumptions as Theorem 2.2, the MLMC finite element solution of (2.5)-(2.6) satisfies the following error estimate:

$$
\begin{aligned}
& \left\|\mathbb{E}\left[\boldsymbol{E}\left(t_{k}\right)\right]-E^{M L}\left[\boldsymbol{E}_{h}^{k}\right]\right\|_{\boldsymbol{L}_{(D \times U)}^{2}}+\left\|\mathbb{E}\left[\boldsymbol{H}\left(t_{k}\right)\right]-E^{M L}\left[\boldsymbol{H}_{h}^{k}\right]\right\|_{\boldsymbol{L}^{2}(D \times U)} \\
\leq & C\left(\tau_{L}^{2}+h_{L}^{r}+\sum_{l=1}^{L}\left(h_{l}^{r}+\tau_{l}^{2}\right) M_{l}^{-1 / 2}\right) .
\end{aligned}
$$

Proof. Similar to the proof of Theorem 2.2, we rewrite the error of $E$ in two parts:

$$
\begin{aligned}
& \left\|\mathbb{E}\left[\boldsymbol{E}\left(t_{k}\right)\right]-E^{M L}\left[\boldsymbol{E}_{h}^{k}\right]\right\|_{\boldsymbol{L}^{2}(D \times U)} \\
= & \left\|\mathbb{E}\left[\boldsymbol{E}\left(t_{k}\right)\right]-\mathbb{E}\left[\boldsymbol{E}_{L}^{k}\right]+\mathbb{E}\left[\boldsymbol{E}_{L}^{k}\right]-\sum_{l=1}^{L} E_{M_{l}}\left[\boldsymbol{E}_{l}^{k}-\boldsymbol{E}_{l-1}^{k}\right]\right\|_{\boldsymbol{L}^{2}(D \times U)} \\
\leq & \left\|\mathbb{E}\left[\boldsymbol{E}\left(t_{k}\right)\right]-\mathbb{E}\left[\boldsymbol{E}_{L}^{k}\right]\right\| \\
\boldsymbol{L}^{2}(D \times U) & +\left\|\sum_{l=1}^{L}\left(\mathbb{E}\left[\boldsymbol{E}_{l}^{k}-\boldsymbol{E}_{l-1}^{k}\right]-E_{M_{l}}\left[\boldsymbol{E}_{l}^{k}-\boldsymbol{E}_{l-1}^{k}\right]\right)\right\|_{\boldsymbol{L}_{(D \times U)}} \\
\leq & \mathbb{E}\left[\left\|\boldsymbol{E}\left(t_{k}\right)-\boldsymbol{E}_{L}^{k}\right\|_{\boldsymbol{L}^{2}(D)}^{2}\right]^{1 / 2}+\left\|\sum_{l=1}^{L}\left(\mathbb{E}\left[\boldsymbol{E}_{l}^{k}-\boldsymbol{E}_{l-1}^{k}\right]-E_{M_{l}}\left[\boldsymbol{E}_{l}^{k}-\boldsymbol{E}_{l-1}^{k}\right]\right)\right\|_{\boldsymbol{L}_{(D \times U)}^{2}} .
\end{aligned}
$$

Similar estimate holds true for $\boldsymbol{H}$. 
Hence, we have

$$
\left\|\mathbb{E}\left[\boldsymbol{E}\left(t_{k}\right)\right]-E^{M L}\left[\boldsymbol{E}_{h}^{k}\right]\right\|_{\boldsymbol{L}^{2}(D \times U)}+\left\|\mathbb{E}\left[\boldsymbol{H}\left(t_{k}\right)\right]-E^{M L}\left[\boldsymbol{H}_{h}^{k}\right]\right\|_{\boldsymbol{L}^{2}(D \times U)} \leq \sqrt{2} I+I I,
$$

where we denote

$$
\begin{aligned}
I:= & \mathbb{E}\left[\left\|\boldsymbol{E}\left(t_{k}\right)-\boldsymbol{E}_{L}^{k}\right\|_{\boldsymbol{L}_{2}(D)}^{2}+\left\|\boldsymbol{H}\left(t_{k}\right)-\boldsymbol{H}_{L}^{k}\right\|_{\boldsymbol{L}_{2}(D)}^{2}\right]^{1 / 2}, \\
I I:= & \left\|\sum_{l=1}^{L}\left(\mathbb{E}\left[\boldsymbol{E}_{l}^{k}-\boldsymbol{E}_{l-1}^{k}\right]-E_{M_{l}}\left[\boldsymbol{E}_{l}^{k}-\boldsymbol{E}_{l-1}^{k}\right]\right)\right\|_{\boldsymbol{L}_{(D \times U)}^{2}} \\
& +\left\|\sum_{l=1}^{L}\left(\mathbb{E}\left[\boldsymbol{H}_{l}^{k}-\boldsymbol{H}_{l-1}^{k}\right]-E_{M_{l}}\left[\boldsymbol{H}_{l}^{k}-\boldsymbol{H}_{l-1}^{k}\right]\right)\right\|_{\boldsymbol{L}_{(D \times U)}^{2}} .
\end{aligned}
$$

The error term $I$ is the error caused by the finite element scheme, which is given by Theorem 2.1.

To estimate term $I I$, by Lemma 2.2 , for any $1 \leq l \leq M$ we have:

$$
\begin{aligned}
& \left\|\left(\mathbb{E}\left[\boldsymbol{E}_{l}^{k}-\boldsymbol{E}_{l-1}^{k}\right]-E_{M_{l}}\left[\boldsymbol{E}_{l}^{k}-\boldsymbol{E}_{l-1}^{k}\right]\right)\right\|_{\boldsymbol{L}^{2}(D \times U)} \\
& +\left\|\left(\mathbb{E}\left[\boldsymbol{H}_{l}^{k}-\boldsymbol{H}_{l-1}^{k}\right]-E_{M_{l}}\left[\boldsymbol{H}_{l}^{k}-\boldsymbol{H}_{l-1}^{k}\right]\right)\right\|_{\boldsymbol{L}^{2}(D \times U)} \\
=\left\|\left(\mathbb{E}-E_{\left.M_{l}\right)}\right)\left[\boldsymbol{E}_{l}^{k}-\boldsymbol{E}_{l-1}^{k}\right]\right\|_{\boldsymbol{L}^{2}(D \times U)}+\left\|\left(\mathbb{E}-E_{\left.M_{l}\right)}\right)\left[\boldsymbol{H}_{l}^{k}-\boldsymbol{H}_{l-1}^{k}\right]\right\|_{\boldsymbol{L}^{2}(D \times U)} & \\
\leq M_{l}^{-\frac{1}{2}}\left(\left\|\boldsymbol{E}^{k}-\boldsymbol{E}_{l}^{k}\right\|_{\boldsymbol{L}^{2}(D \times U)}+\left\|\boldsymbol{E}^{k}-\boldsymbol{E}_{l-1}^{k}\right\|_{\boldsymbol{L}^{2}(D \times U)}\right. & \left.\quad\left\|\boldsymbol{H}^{k}-\boldsymbol{H}_{l}^{k}\right\|_{\boldsymbol{L}^{2}(D \times U)}+\left\|\boldsymbol{H}^{k}-\boldsymbol{H}_{l-1}^{k}\right\|_{\boldsymbol{L}^{2}(D \times U)}\right) \\
\leq & C M_{l}^{-\frac{1}{2}}\left(h_{l}^{r}+\tau_{l}^{2}+h_{l-1}^{r}+\tau_{l-1}^{2}\right) \leq C M_{l}^{-\frac{1}{2}}\left(h_{l}^{r}+\tau_{l}^{2}\right) .
\end{aligned}
$$

Hence we have the error estimate for term $I I: I I \leq C \sum_{l=1}^{L}\left(h_{l}^{r}+\tau_{l}^{2}\right) M_{l}^{-\frac{1}{2}}$, which, along with the estimate of $I$, completes the proof.

\section{Quasi-Monte Carlo finite element method}

Due to the slow convergence of the classical Monte Carlo method, the quasi-Monte Carlo methods have been proposed to solve stochastic elliptic equations (e.g., [11,29]). In this section, we will analyze the usage of this method for solving the stochastic Maxwell's equations. Instead of considering the expectation of $\boldsymbol{E}$ and $\boldsymbol{H}$ directly, we will find out estimator of $\mathbb{E}\left[G_{1}(\boldsymbol{E})\right]$ and $\mathbb{E}\left[G_{2}(\boldsymbol{H})\right]$ respectively, where $G_{1}, G_{2}: L^{2}(D) \mapsto \mathbb{R}$ are some bounded linear functionals. 


\subsection{QMC integration in the finite dimensional setting}

For any function $F$ defined on $U=[0,1]^{n}$, consider the following integral

$$
I(F):=\int_{U} F(\boldsymbol{y}) d \boldsymbol{y} .
$$

To approximate $I(F)$, we use the $N$ point QMC estimator given by

$$
Q_{N}(F):=\frac{1}{N} \sum_{i=1}^{N} F\left(\boldsymbol{y}^{(i)}\right),
$$

where $\left\{\boldsymbol{y}^{(i)}\right\}_{i=1}^{N} \subset U$ is the set of points which needs to be chosen carefully. Here we just focus on the shifted rank-1 lattice rules. In these rules, the quadrature points are given by the following formula

$$
\boldsymbol{y}^{(i)}=\operatorname{frac}\left(\frac{i \mathbf{z}}{N}+\boldsymbol{\Delta}\right), \quad i=1,2, \cdots N,
$$

where $\mathbf{z} \in \mathbb{Z}^{s}$ is known as the generating vector, $\Delta \in[0,1]^{s}$ is the shift, and frac $(\cdot)$ means taking the fractional part of each component in the vector. More details on the general theory and choices of quadrature points for QMC lattice rules for the s-dimensional cube can be found in $[10,11]$ and references therein.

To measure the error of this method, we need the following weighted and unanchored Sobolev space $\mathcal{W}_{s, \gamma}$ which is a Hilbert space containing functions defined over $U$, equipped with the norm

$$
\begin{aligned}
& \|F\|_{\mathcal{W}_{s, \gamma}}^{2}:=\sum_{\mathfrak{u} \subset\{1: s\}} \gamma_{\mathfrak{u}}^{-1} \int_{[0,1]^{|\mathfrak{u}|}}\left(\int_{[0,1]^{s-|u|} \mid} \frac{\partial^{|\mathfrak{u}|} F}{\partial \boldsymbol{y}_{\mathfrak{u}}}\left(\boldsymbol{y}_{\mathfrak{u}} ; \boldsymbol{y}_{\{1: s\} \backslash \mathfrak{u}}\right) d \boldsymbol{y}_{\{1: s\} \backslash \mathfrak{u}}\right)^{2} d \boldsymbol{y}_{\mathfrak{u}} \\
& =\sum_{\mathfrak{u} \subseteq\{1: s\}} \gamma_{\mathfrak{u}}^{-1} \int_{[0,1]^{|\mathfrak{u}|}}\left(\int_{[0,1]^{s-|u|}} \partial^{\mathfrak{u}} F\left(\boldsymbol{y}_{\mathfrak{u}} ; \boldsymbol{y}_{\{1: s\} \backslash \mathfrak{u}}\right) d \boldsymbol{y}_{\{1: s\} \backslash \mathfrak{u}}\right)^{2} d \boldsymbol{y}_{\mathfrak{u}^{\prime}}
\end{aligned}
$$

where $\{1: s\}$ is a shorthand notation for the set of indices $\{1,2, \cdots, s\}, \frac{\partial^{|\mathfrak{u}|} F}{\partial \boldsymbol{y}_{u}}$ and $\partial^{\mathfrak{u}} F$ denote the mixed first derivative with respect to the active variables $y_{j}$ with $j \in \mathfrak{u}$, and $\boldsymbol{y}_{\{1: s\} \backslash \mathfrak{u}}$ denotes the inactive variables $y_{j}$ with $j \notin \mathfrak{u}$. And $\gamma_{\mathfrak{u}} \geq 0$ is a weight parameter associated with each group of variables $y_{\mathfrak{u}}$, with the convention that $\gamma_{\varnothing}=1$. If $\gamma_{\mathfrak{u}}=0$, then we expect that the corresponding integral of the mixed first derivative is also zero, and we follow the convention " $0 / 0=0 "$.

The weighted spaces was first introduced by Sloan and Woźniakowski [40] and later generalized in many papers (e.g., [12,39]). We now state the essential theorem for QMC error estimate. 
Theorem 3.1. [28, Theorem 4.1] Let $s, N \in \mathbb{N}$ be given, and assume $F \in \mathcal{W}_{s, \gamma}$ for a particular choice of weights $\gamma$. Then a randomly shifted lattice rule can be constructed using a componentby-component algorithm such that the root-mean-square error satisfies: for all $\lambda \in(1 / 2,1]$,

$$
\sqrt{\mathbb{E}\left[\left|I(F)-Q_{N}(F)\right|^{2}\right]} \leq\left(\sum_{\varnothing \neq \mathfrak{u} \subseteq\{1: s\}} \gamma_{\mathfrak{u}}^{\lambda}\left(\frac{2 \zeta(2 \lambda)}{\left(2 \pi^{2}\right)^{\lambda}}\right)^{|\mathfrak{u}|}\right)^{\frac{1}{2 \lambda}} \varphi(N)^{-\frac{1}{2 \lambda}\|F\|_{\mathcal{W}_{s, \gamma}}}
$$

where $\mathbb{E}[\cdot]$ denotes the expectation with respect to the random shift which is uniformly distributed over $U, \zeta(\cdot)$ is the Riemann zeta function, and $\varphi(N)$ is the Euler totient function [28].

\subsection{Regularity analysis with respect to the random vector}

To obtain the error estimate for the QMC finite element method, we need the regularity estimate for the solution of (1.1)-(1.4) with respect to the random vector.

First, we have the following energy conservation property.

Theorem 3.2. For the solution $(\boldsymbol{E}, \boldsymbol{H})$ of (1.1)-(1.4), we have: $\forall m \geq 0$ and $\forall t \in[0, T]$,

$$
\left(\left\|\epsilon^{\frac{1}{2}} \partial_{t}^{m} \boldsymbol{E}\right\|_{\boldsymbol{L}^{2}(D \times U)}^{2}+\left\|\mu^{\frac{1}{2}} \partial_{t}^{m} \boldsymbol{H}\right\|_{\boldsymbol{L}^{2}(D \times U)}^{2}\right)(t)=\left(\left\|\epsilon^{\frac{1}{2}} \partial_{t}^{m} \boldsymbol{E}\right\|_{\boldsymbol{L}^{2}(D \times U)}^{2}+\left\|\mu^{\frac{1}{2}} \partial_{t}^{m} \boldsymbol{H}\right\|_{\boldsymbol{L}^{2}(D \times U)}^{2}\right)(0),
$$

here and below we denote $\partial_{t}^{m}:=\partial_{t^{m}}$ for the $m$-th derivative with respect to variable $t$.

Proof. When $m=0$, the proof is the same as that of [30, Lemma 2.1] even when $\epsilon$ and $\mu$ depend on spatial variable $x$.

For any $m \geq 1$, taking the $m$-th time derivative of (1.1) and (1.2), multiplying the respective result by $\partial_{t}^{m} \boldsymbol{E}$ and $\partial_{t}^{m} \boldsymbol{H}$, then integrating over $\Omega$ and $D$, and adding the results together, we obtain

$$
\begin{aligned}
& \frac{1}{2} \frac{d}{d t}\left(\left\|\epsilon^{\frac{1}{2}} \partial_{t}^{m} \boldsymbol{E}\right\|_{\boldsymbol{L}^{2}(D \times U)}^{2}+\left\|\mu^{\frac{1}{2}} \partial_{t}^{m} \boldsymbol{H}\right\|_{\boldsymbol{L}^{2}(D \times U)}^{2}\right) \\
= & \int_{U} \int_{D} \nabla \times \partial_{t}^{m} \boldsymbol{H} \cdot \partial_{t}^{m} \boldsymbol{E} d \mathbf{x} d \boldsymbol{y}-\int_{U} \int_{D} \nabla \times \partial_{t}^{m} \boldsymbol{E} \cdot \partial_{t}^{m} \boldsymbol{H} d \mathbf{x} d \boldsymbol{y} \\
= & -\int_{U} \int_{\partial D}\left(\mathbf{n} \times \partial_{t}^{m} \boldsymbol{E}\right) \cdot \partial_{t}^{m} \boldsymbol{H} d s d \boldsymbol{y} \\
= & -\int_{U} \int_{\partial D} \partial_{t}^{m}(\mathbf{n} \times \boldsymbol{E}) \cdot \partial_{t^{m}} \boldsymbol{H} d s d \boldsymbol{y}=0,
\end{aligned}
$$

where we used the PEC boundary condition (1.3) in the last step. Integrating (3.2) from $t=0$ to any time $t$ concludes the proof.

With Theorem 3.2, we can prove the following bound for the first derivative of the solution with respect to the random vector. 
Theorem 3.3. Denote constant $C_{s}^{\epsilon \mu}:=\left|\epsilon^{-1} \partial_{y_{i}}^{s} \epsilon\right|_{L^{\infty}(D \times U)}+\left|\mu^{-1} \partial_{y_{i}}^{s} \mu\right|_{L^{\infty}(D \times U)}$ for any integer $s \geq 1$. Then for solution $(\boldsymbol{E}, \boldsymbol{H})$ of (1.1)-(1.4), we have: for any $t \in[0, T]$ and $y_{i}$,

$$
\begin{aligned}
& \left(\left\|\epsilon^{\frac{1}{2}} \partial_{y_{i}} \boldsymbol{E}\right\|_{\boldsymbol{L}^{2}(D \times U)}^{2}+\left\|\mu^{\frac{1}{2}} \partial_{y_{i}} \boldsymbol{H}\right\|_{\boldsymbol{L}^{2}(D \times U)}^{2}\right)(t) \\
\leq & {\left[t C_{1}^{\epsilon \mu}\left(\left\|\epsilon^{\frac{1}{2}} \partial_{t} \boldsymbol{E}\right\|_{\boldsymbol{L}^{2}(D \times U)}^{2}+\left\|\mu^{\frac{1}{2}} \partial_{t} \boldsymbol{H}\right\|_{\boldsymbol{L}^{2}(D \times U)}^{2}\right)(0)\right.} \\
& \left.+\left(\left\|\epsilon^{\frac{1}{2}} \partial_{y_{i}} \boldsymbol{E}\right\|_{\boldsymbol{L}_{(D \times U)}^{2}}^{2}+\left\|\mu^{\frac{1}{2}} \partial_{y_{i}} \boldsymbol{H}\right\|_{\boldsymbol{L}_{(D \times U)}^{2}}^{2}\right)(0)\right] \exp \left(t C_{1}^{\epsilon \mu}\right) .
\end{aligned}
$$

Proof. Differentiating (1.1) and (1.2) with respect to $y_{i}$, respectively, we have

$$
\begin{aligned}
& \epsilon \partial_{t} \partial_{y_{i}} \boldsymbol{E}+\partial_{y_{i}} \epsilon \cdot \partial_{t} \boldsymbol{E}=\nabla \times \partial_{y_{i}} \boldsymbol{H}, \\
& \mu \partial_{t} \partial_{y_{i}} \boldsymbol{H}+\partial_{y_{i}} \mu \cdot \partial_{t} \boldsymbol{H}=-\nabla \times \partial_{y_{i}} \boldsymbol{E} .
\end{aligned}
$$

Multiplying (3.3) and (3.4) by $\partial_{y_{i}} E$ and $\partial_{y_{i}} H$, respectively, then integrating over $U$ and $D$, and adding the resultants together, we obtain

$$
\begin{aligned}
& \frac{1}{2} \frac{d}{d t}\left(\left\|\epsilon^{\frac{1}{2}} \partial_{y_{i}} \boldsymbol{E}\right\|_{\mathbf{L}^{2}(D \times U)}^{2}+\left\|\mu^{\frac{1}{2}} \partial_{y_{i}} \boldsymbol{H}\right\|_{\mathbf{L}^{2}(D \times U)}^{2}\right) \\
= & \int_{U} \int_{D}\left(-\partial_{y_{i}} \epsilon \cdot \partial_{t} \boldsymbol{E}+\nabla \times \partial_{y_{i}} \boldsymbol{H}\right) \cdot \partial_{y_{i}} \boldsymbol{E} d \mathbf{x} d \mathbf{y}-\int_{U} \int_{D}\left(\partial_{y_{i}} \mu \cdot \partial_{t} \boldsymbol{H}+\nabla \times \partial_{y_{i}} \boldsymbol{E}\right) \cdot \partial_{y_{i}} \boldsymbol{H} d \mathbf{x} d \mathbf{y} \\
= & -\int_{U} \int_{D} \partial_{y_{i}} \epsilon \cdot \partial_{t} \boldsymbol{E} \cdot \partial_{y_{i}} \boldsymbol{E} d \mathbf{x} d \mathbf{y}-\int_{U} \int_{D} \partial_{y_{i}} \mu \cdot \partial_{t} \boldsymbol{H} \cdot \partial_{y_{i}} \boldsymbol{H} d \mathbf{x} d \mathbf{y},
\end{aligned}
$$

where we used integration by parts and the PEC boundary condition (1.3) in the last step, i.e.,

$$
\int_{U} \int_{D} \nabla \times \partial_{y_{i}} \boldsymbol{E} \cdot \partial_{y_{i}} \boldsymbol{H}=\int_{U} \int_{\partial D} \boldsymbol{n} \times \partial_{y_{i}} \boldsymbol{E} \cdot \partial_{y_{i}} \boldsymbol{H}+\int_{U} \int_{D} \partial_{y_{i}} \boldsymbol{E} \cdot \nabla \times \partial_{y_{i}} \boldsymbol{H}=\int_{U} \int_{D} \partial_{y_{i}} \boldsymbol{E} \cdot \nabla \times \partial_{y_{i}} \boldsymbol{H} .
$$

By the Cauchy-Schwarz inequality, from (3.5) we have

$$
\begin{aligned}
& \quad \frac{1}{2} \frac{d}{d t}\left(\left\|\epsilon^{\frac{1}{2}} \partial_{y_{i}} E\right\|_{L^{2}(D \times U)}^{2}+\left\|\mu^{\frac{1}{2}} \partial_{y_{i}} H\right\|_{L^{2}(D \times U)}^{2}\right) \\
& \leq\left|\epsilon^{-1} \partial_{y_{i}} \epsilon\right|_{L^{\infty}(D \times U)} \frac{1}{2}\left(\left\|\epsilon^{\frac{1}{2}} \partial_{t} E\right\|_{L^{2}(D \times U)}^{2}+\left\|\epsilon^{\frac{1}{2}} \partial_{y_{i}} E\right\|_{\left.L_{(D \times U)}^{2}\right)}^{2}\right) \\
& \quad+\left|\mu^{-1} \partial_{y_{i}} \mu\right|_{L^{\infty}(D \times U)} \frac{1}{2}\left(\left\|\mu^{\frac{1}{2}} \partial_{t} \boldsymbol{H}\right\|_{L^{2}(D \times U)}^{2}+\left\|\mu^{\frac{1}{2}} \partial_{y_{i}} \boldsymbol{H}\right\|_{L^{2}(D \times U)}^{2}\right) \\
& \leq C_{1}^{\epsilon \mu} \cdot \frac{1}{2}\left(\left\|\epsilon^{\frac{1}{2}} \partial_{t} E\right\|_{L^{2}(D \times U)}^{2}+\left\|\mu^{\frac{1}{2}} \partial_{t} H\right\|_{L^{2}(D \times U)}^{2}\right)(0) \\
& \quad+C_{1}^{\epsilon \mu} \cdot \frac{1}{2}\left(\left\|\epsilon^{\frac{1}{2}} \partial_{y_{i}} E\right\|_{L_{(D \times U)}^{2}}^{2}+\left\|\mu^{\frac{1}{2}} \partial_{y_{i}} H\right\|_{L_{(D \times U)}^{2}}^{2}\right)
\end{aligned}
$$

where we used the notation of $C_{1}^{\epsilon \mu}$ and Theorem 3.2 in the last step.

Using the Gronwall inequality to the last inequality concludes the proof. 
By the same technique, we can prove the following bound for the higher order derivatives of the solution with respect to the random vector.

Theorem 3.4. For the solution $(\boldsymbol{E}, \boldsymbol{H})$ of (1.1)-(1.4) and any $|\boldsymbol{m}| \geq 1$, we have: $\forall t \in[0, T]$,

$$
\begin{aligned}
& \left(\left\|\epsilon^{\frac{1}{2}} \partial_{\boldsymbol{y}}^{|\boldsymbol{m}|} \boldsymbol{E}\right\|_{\boldsymbol{L}_{(D \times U)}^{2}}^{2}+\left\|\mu^{\frac{1}{2}} \partial_{\boldsymbol{y}}^{|\boldsymbol{m}|} \boldsymbol{H}\right\|_{\boldsymbol{L}^{2}(D \times U)}^{2}\right)(t) \\
\leq & \exp \left(t \sum_{1 \leq|\boldsymbol{S}| \leq|\boldsymbol{m}|}\left(\begin{array}{c}
\boldsymbol{m} \\
\boldsymbol{s}
\end{array}\right) C_{s}^{\epsilon \mu}\right) \cdot\left[\left(\left\|\epsilon^{\frac{1}{2}} \partial_{\boldsymbol{y}}^{|\boldsymbol{m}|} \boldsymbol{E}\right\|_{\boldsymbol{L}^{2}(D \times U)}^{2}+\left\|\mu^{\frac{1}{2}} \partial_{\boldsymbol{y}}^{|\boldsymbol{m}|} \boldsymbol{H}\right\|_{\boldsymbol{L}^{2}(D \times U)}^{2}\right)(0)\right. \\
& \left.+\int_{0}^{t} \sum_{1 \leq|\boldsymbol{S}| \leq|\boldsymbol{m}|}\left(\begin{array}{c}
\boldsymbol{m} \\
\boldsymbol{s}
\end{array}\right) C_{s}^{\epsilon \mu}\left(\left\|\epsilon^{\frac{1}{2}} \partial_{\boldsymbol{y}}^{|\boldsymbol{m}|-|\boldsymbol{S}|} \partial_{t} \boldsymbol{E}\right\|_{\boldsymbol{L}_{(D \times U)}^{2}}^{2}+\left\|\mu^{\frac{1}{2}} \partial_{\boldsymbol{y}}^{|\boldsymbol{m}|-|\boldsymbol{S}|} \partial_{t} \boldsymbol{H}\right\|_{\boldsymbol{L}^{2}(D \times U)}^{2}\right) d t\right],
\end{aligned}
$$

where we denote $\partial_{\boldsymbol{y}}^{|\boldsymbol{m}|}=\partial_{y_{1}}^{m_{1}} \cdots \partial_{y_{n}}^{m_{n}}$ and $\left(\begin{array}{c}\boldsymbol{m} \\ \boldsymbol{s}\end{array}\right)=\prod_{j=1}^{n}\left(\begin{array}{c}m_{j} \\ s_{j}\end{array}\right)$ for any $\boldsymbol{m}=\left(m_{1}, \cdots, m_{n}\right)$ and $\boldsymbol{s}=$ $\left(s_{1}, \cdots, s_{n}\right)$ with $m_{i}$ and $s_{i}$ either 0 or 1 .

Proof. Taking the $|m|$-th mixed derivative of (1.1) and (1.2) with respect to $y_{1}, \cdots, y_{n}$, respectively, we have

$$
\begin{aligned}
& \epsilon \partial_{t}\left(\partial_{\boldsymbol{y}}^{|\boldsymbol{m}|} \boldsymbol{E}\right)=\nabla \times \partial_{\boldsymbol{y}}^{|\boldsymbol{m}|} \boldsymbol{H}-\sum_{1 \leq|\boldsymbol{S}| \leq|\boldsymbol{m}|}\left(\begin{array}{c}
\boldsymbol{m} \\
\boldsymbol{s}
\end{array}\right) \partial_{\boldsymbol{y}}^{|\boldsymbol{S}|} \epsilon \partial_{\boldsymbol{y}}^{|\boldsymbol{m}|-|\boldsymbol{S}|} \partial_{t} \boldsymbol{E} \\
& \mu \partial_{t}\left(\partial_{\boldsymbol{y}}^{|\boldsymbol{m}|} \boldsymbol{H}\right)=-\nabla \times \partial_{\boldsymbol{y}}^{|\boldsymbol{m}|} \boldsymbol{E}-\sum_{1 \leq|\boldsymbol{S}| \leq|\boldsymbol{m}|}\left(\begin{array}{c}
\boldsymbol{m} \\
\boldsymbol{s}
\end{array}\right) \partial_{\boldsymbol{y}}^{|\boldsymbol{S}|} \mu \partial_{\boldsymbol{y}}^{|\boldsymbol{m}|-|\boldsymbol{S}|} \partial_{t} \boldsymbol{H} .
\end{aligned}
$$

Multiplying (3.6) and (3.7) by $\partial_{\boldsymbol{y}}^{|\boldsymbol{m}|} E$ and $\partial_{\boldsymbol{y}}^{|\boldsymbol{m}|} \boldsymbol{H}$, respectively, then integrating over $U$ and $D$, and adding the resultants together, we easily obtain

$$
\begin{aligned}
& \frac{1}{2} \frac{d}{d t}\left(\left\|\epsilon^{\frac{1}{2}} \partial_{\boldsymbol{y}}^{|\boldsymbol{m}|} \boldsymbol{E}\right\|_{\boldsymbol{L}_{(D \times U)}^{2}}^{2}+\left\|\mu^{\frac{1}{2}} \partial_{\boldsymbol{y}}^{|\boldsymbol{m}|} \boldsymbol{H}\right\|_{L^{2}(D \times U)}^{2}\right) \\
& =-\int_{U} \int_{D_{1 \leq|\boldsymbol{S}| \leq|\boldsymbol{m}|}}\left(\begin{array}{c}
\boldsymbol{m} \\
\boldsymbol{s}
\end{array}\right) \partial_{\boldsymbol{y}}^{|\boldsymbol{S}|} \epsilon \partial_{\boldsymbol{y}}^{|\boldsymbol{m}|-|\boldsymbol{S}|} \partial_{t} E \cdot \partial_{\boldsymbol{y}}^{|\boldsymbol{m}|} E d \mathbf{x} d \mathbf{y} \\
& -\int_{U} \int_{D_{1 \leq|\boldsymbol{S}| \leq|\boldsymbol{m}|}}\left(\begin{array}{c}
\boldsymbol{m} \\
\boldsymbol{s}
\end{array}\right) \partial_{\boldsymbol{y}}^{|\boldsymbol{S}|} \mu \partial_{\boldsymbol{y}}^{|\boldsymbol{m}|-|\boldsymbol{S}|} \partial_{t} \boldsymbol{H} \cdot \partial_{\mathbf{y}}^{m} \boldsymbol{H} d \mathbf{x} d \mathbf{y} \\
& \leq \sum_{1 \leq|\boldsymbol{S}| \leq|\boldsymbol{m}|}\left(\begin{array}{c}
\boldsymbol{m} \\
\boldsymbol{s}
\end{array}\right) C_{s}^{\epsilon \mu} \cdot \frac{1}{2}\left(\left\|\epsilon^{\frac{1}{2}} \partial_{\boldsymbol{y}}^{|\boldsymbol{m}|-|\boldsymbol{s}|} \partial_{t} \boldsymbol{E}\right\|_{\boldsymbol{L}^{2}(D \times U)}^{2}+\left\|\mu^{\frac{1}{2}} \partial_{\boldsymbol{y}}^{|\boldsymbol{m}|-|\boldsymbol{s}|} \partial_{t} \boldsymbol{H}\right\|_{\boldsymbol{L}^{2}(D \times U)}^{2}\right) \\
& +\sum_{1 \leq|\boldsymbol{S}| \leq|\boldsymbol{m}|}\left(\begin{array}{c}
\boldsymbol{m} \\
s
\end{array}\right) C_{s}^{\epsilon \mu} \cdot \frac{1}{2}\left(\left\|e^{\frac{1}{2}} \partial_{\boldsymbol{y}}^{|\boldsymbol{m}|} \boldsymbol{E}\right\|_{\boldsymbol{L}^{2}(D \times U)}^{2}+\left\|\mu^{\frac{1}{2}} \partial_{\boldsymbol{y}}^{|\boldsymbol{m}|} \boldsymbol{H}\right\|_{\boldsymbol{L}^{2}(D \times U)}^{2}\right),
\end{aligned}
$$

which, along with the Gronwall inequality, completes the proof. 
Note that Theorem 3.4 involves the estimate $\left\|\epsilon^{\frac{1}{2}} \partial_{\boldsymbol{y}}^{|\boldsymbol{m}|-|\boldsymbol{S}|} \partial_{t} \boldsymbol{E}\right\|_{L^{2}(D \times U)}^{2}$ and $\left\|\mu^{\frac{1}{2}} \partial_{\boldsymbol{y}}^{|\boldsymbol{m}|-|\boldsymbol{S}|} \partial_{t} \boldsymbol{H}\right\|_{L^{2}(D \times U)}^{2}$, which can be bounded as below.

Theorem 3.5. For the solution $(\boldsymbol{E}, \boldsymbol{H})$ of (1.1)-(1.4) and any $|\boldsymbol{m}|, n \geq 1$, we have: $\forall t \in[0, T]$,

$$
\begin{aligned}
& \left(\left\|\epsilon^{\frac{1}{2}} \partial_{\boldsymbol{y}}^{|\boldsymbol{m}|} \partial_{t}^{n} \boldsymbol{E}\right\|_{\boldsymbol{L}_{(D \times U)}^{2}}^{2}+\left\|\mu^{\frac{1}{2}} \partial_{\boldsymbol{y}}^{|\boldsymbol{m}|} \partial_{t}^{n} \boldsymbol{H}\right\|_{\boldsymbol{L}^{2}(D \times U)}^{2}\right)(t) \\
\leq & \exp \left(t \sum_{1 \leq|\boldsymbol{S}| \leq|\boldsymbol{m}|}\left(\begin{array}{c}
\boldsymbol{m} \\
\boldsymbol{s}
\end{array}\right) C_{s}^{\epsilon \mu}\right) \cdot\left[\left(\left\|\epsilon^{\frac{1}{2}} \partial_{\boldsymbol{y}}^{|\boldsymbol{m}|} \partial_{t}^{n} \boldsymbol{E}\right\|_{\boldsymbol{L}^{2}(D \times U)}^{2}+\left\|\mu^{\frac{1}{2}} \partial_{\boldsymbol{y}}^{|\boldsymbol{m}|} \partial_{t}^{n} \boldsymbol{H}\right\|_{\boldsymbol{L}^{2}(D \times U)}^{2}\right)(0)\right. \\
& \left.+\int_{0}^{t} \sum_{1 \leq|\boldsymbol{S}| \leq|\boldsymbol{m}|}\left(\begin{array}{c}
\boldsymbol{m} \\
\boldsymbol{s}
\end{array}\right) C_{s}^{\epsilon \mu}\left(\left\|\epsilon^{\frac{1}{2}} \partial_{\boldsymbol{y}}^{|\boldsymbol{m}|-|\boldsymbol{s}|} \partial_{t}^{n+1} \boldsymbol{E}\right\|_{\boldsymbol{L}_{(D \times U)}^{2}}^{2}+\left\|\mu^{\frac{1}{2}} \partial_{\boldsymbol{y}}^{|\boldsymbol{m}|-|\boldsymbol{S}|} \partial_{t}^{n+1} \boldsymbol{H}\right\|_{\boldsymbol{L}_{(D \times U)}^{2}}^{2}\right) d t\right] .
\end{aligned}
$$

Proof. Taking the $n$-th derivative of (3.6) and (3.7) with respect to $t$, respectively, we have

$$
\begin{aligned}
& \epsilon \partial_{t}\left(\partial_{\boldsymbol{y}}^{|\boldsymbol{m}|} \partial_{t}^{n} \boldsymbol{E}\right)=\nabla \times \partial_{\boldsymbol{y}}^{|\boldsymbol{m}|} \partial_{t}^{n} \boldsymbol{H}-\sum_{1 \leq|\boldsymbol{S}| \leq|\boldsymbol{m}|}\left(\begin{array}{c}
\boldsymbol{m} \\
\boldsymbol{s}
\end{array}\right) \partial_{\boldsymbol{y}}^{|\boldsymbol{S}|} \epsilon \partial_{\boldsymbol{y}}^{|\boldsymbol{m}|-|\boldsymbol{s}|} \partial_{t}^{n+1} \boldsymbol{E}, \\
& \mu \partial_{t}\left(\partial_{\boldsymbol{y}}^{|\boldsymbol{m}|} \partial_{t}^{n} \boldsymbol{H}\right)=-\nabla \times \partial_{\boldsymbol{y}}^{|\boldsymbol{m}|} \partial_{t}^{n} \boldsymbol{E}-\sum_{1 \leq|\boldsymbol{S}| \leq|\boldsymbol{m}|}\left(\begin{array}{c}
\boldsymbol{m} \\
\boldsymbol{s}
\end{array}\right) \partial_{\boldsymbol{y}}^{|\boldsymbol{S}|} \mu \partial_{\boldsymbol{y}}^{|\boldsymbol{m}|-|\boldsymbol{S}|} \partial_{t}^{n+1} \boldsymbol{H} .
\end{aligned}
$$

Multiplying (3.9) and (3.10) by $\partial_{y}^{|\boldsymbol{m}|} \partial_{t}^{n} \boldsymbol{E}$ and $\partial_{\boldsymbol{y}}^{|\boldsymbol{m}|} \partial_{t}^{n} \boldsymbol{H}$, respectively, then following the proof of Theorem 3.4, we easily conclude the proof.

Using Theorem 3.2, and Theorem 3.5 recursively in Theorem 3.4, we can see that the higher order derivatives $\left(\left\|\epsilon^{\frac{1}{2}} \partial_{\boldsymbol{y}}^{|\boldsymbol{m}|} \boldsymbol{E}\right\|_{\boldsymbol{L}_{(D \times U)}^{2}}^{2}+\left\|\mu^{\frac{1}{2}} \partial_{\boldsymbol{y}}^{|\boldsymbol{m}|} \boldsymbol{H}\right\|_{\boldsymbol{L}_{(D \times U)}^{2}}^{2}\right)(t)$ can be bounded by a linear combination of the initial values:

$$
\begin{aligned}
& \left\|\epsilon^{\frac{1}{2}} \partial_{\boldsymbol{y}}^{|\boldsymbol{m}|} \boldsymbol{E}\right\|_{\boldsymbol{L}_{(D \times U)}^{2}}^{2}(0),\left\|\mu^{\frac{1}{2}} \partial_{\boldsymbol{y}}^{|\boldsymbol{m}|} \boldsymbol{H}\right\|_{\boldsymbol{L}_{(D \times U)}^{2}}^{2}(0), \\
& \left\|\partial_{\boldsymbol{y}}^{|\boldsymbol{S}|} \partial_{t}^{l} \boldsymbol{E}\right\|_{\boldsymbol{L}_{(D \times U)}^{2}}^{2}(0), \quad\left\|\partial_{\boldsymbol{y}}^{|\boldsymbol{S}|} \partial_{t}^{l} \boldsymbol{H}\right\|_{\boldsymbol{L}_{(D \times U)}^{2}}^{2}(0), \quad \forall 1 \leq l \leq|\boldsymbol{m}|, \quad 1 \leq|\boldsymbol{s}|+l \leq|\boldsymbol{m}| .
\end{aligned}
$$

But an explicit expression for the bound is too complicated to write down due to the recursive dependence. Below we illustrate the exact bound for $m=2$. 
Theorem 3.6. For the solution $(\boldsymbol{E}, \boldsymbol{H})$ of (1.1)-(1.4) and any $t \in[0, T]$, we have:

$$
\begin{aligned}
& \left(\left\|\epsilon^{\frac{1}{2}} \partial_{\boldsymbol{y}}^{2} \boldsymbol{E}\right\|_{\boldsymbol{L}_{(D \times U)}^{2}}^{2}+\left\|\mu^{\frac{1}{2}} \partial_{\boldsymbol{y}}^{2} \boldsymbol{H}\right\|_{\boldsymbol{L}_{(D \times U)}^{2}}^{2}\right)(t) \\
& \leq \exp \left(t\left(2 C_{1}^{\epsilon \mu}+C_{2}^{\epsilon \mu}\right)\right)\left\{\left(\left\|\epsilon^{\frac{1}{2}} \partial_{\boldsymbol{y}}^{2} \boldsymbol{E}\right\|_{\boldsymbol{L}^{2}(D \times U)}^{2}+\left\|\mu^{\frac{1}{2}} \partial_{\boldsymbol{y}}^{2} \boldsymbol{H}\right\|_{\boldsymbol{L}^{2}(D \times U)}^{2}\right)(0)\right. \\
& +2 \exp \left(t C_{1}^{\epsilon \mu}\right)\left[\left(\left\|\epsilon^{\frac{1}{2}} \partial y_{t} \partial_{t}\right\|_{L^{2}(D \times U)}^{2}+\left\|\mu^{\frac{1}{2}} \partial y_{t} \partial_{t}\right\|_{L^{2}(D \times U)}^{2}\right)(0)\right. \\
& \left.+t C_{1}^{\epsilon \mu}\left(\left\|\epsilon^{\frac{1}{2}} \partial_{t}^{2} \boldsymbol{E}\right\|_{\boldsymbol{L}_{(D \times U)}^{2}}^{2}+\left\|\mu^{\frac{1}{2}} \partial_{t}^{2} \boldsymbol{H}\right\|_{\boldsymbol{L}_{(D \times U)}^{2}}^{2}\right)(0)\right] \\
& \left.+t C_{2}^{\epsilon \mu}\left(\left\|\epsilon^{\frac{1}{2}} \partial_{t} \boldsymbol{E}\right\|_{\boldsymbol{L}_{(D \times U)}^{2}}^{2}+\left\|\mu^{\frac{1}{2}} \partial_{t} \boldsymbol{H}\right\|_{\boldsymbol{L}_{(D \times U)}^{2}}^{2}\right)(0)\right\} .
\end{aligned}
$$

Proof. Using Theorem 3.4 for $m=2$, we have

$$
\begin{aligned}
& \left(\left\|\epsilon^{\frac{1}{2}} \partial_{\boldsymbol{y}}^{2} \boldsymbol{E}\right\|_{\boldsymbol{L}^{2}(D \times U)}^{2}+\left\|\mu^{\frac{1}{2}} \partial_{\boldsymbol{y}}^{2} \boldsymbol{H}\right\|_{\boldsymbol{L}^{2}(D \times U)}^{2}\right)(t) \\
& \leq \exp \left(t\left(2 C_{1}^{\epsilon \mu}+C_{2}^{\epsilon \mu}\right)\right) \cdot\left\{\left(\left\|\epsilon^{\frac{1}{2}} \partial_{\mathbf{y}}^{2} \boldsymbol{E}\right\|_{\boldsymbol{L}^{2}(D \times U)}^{2}+\left\|\mu^{\frac{1}{2}} \partial_{\mathbf{y}}^{2} \boldsymbol{H}\right\|_{\boldsymbol{L}_{(D \times U)}^{2}}^{2}\right)(0)\right. \\
& +\int_{0}^{t}\left[2 C_{1}^{\epsilon \mu}\left(\left\|\epsilon^{\frac{1}{2}} \partial_{\boldsymbol{y}} \partial_{t} \boldsymbol{E}\right\|_{\boldsymbol{L}_{(D \times U)}^{2}}^{2}+\left\|\mu^{\frac{1}{2}} \partial_{\boldsymbol{y}} \partial_{t} \boldsymbol{H}\right\|_{\boldsymbol{L}_{(D \times U)}^{2}}^{2}\right)\right. \\
& \left.\left.+C_{2}^{\epsilon \mu}\left(\left\|\epsilon^{\frac{1}{2}} \partial_{t} \boldsymbol{E}\right\|_{L^{2}(D \times U)}^{2}+\left\|\mu^{\frac{1}{2}} \partial_{t} H\right\|_{L^{2}(D \times U)}^{2}\right)\right] d t\right\} .
\end{aligned}
$$

To bound the $\partial_{\boldsymbol{y}} \partial_{t} \boldsymbol{E}$ and $\partial_{\boldsymbol{y}} \partial_{t} \boldsymbol{H}$ terms in (3.13), we use Theorem 3.5 with $m=n=1$ to obtain

$$
\begin{aligned}
& \left(\left\|\epsilon^{\frac{1}{2}} \partial_{\boldsymbol{y}} \partial_{t} \boldsymbol{E}\right\|_{\boldsymbol{L}^{2}(D \times U)}^{2}+\left\|\mu^{\frac{1}{2}} \partial_{\boldsymbol{y}} \partial_{t} \boldsymbol{H}\right\|_{\boldsymbol{L}_{(D \times U)}^{2}}^{2}\right)(t) \\
& \leq \exp \left(t C_{1}^{\epsilon \mu}\right) \cdot\left[\left(\left\|\epsilon^{\frac{1}{2}} \partial_{y} \partial_{t} \boldsymbol{E}\right\|_{L^{2}(D \times U)}^{2}+\left\|\mu^{\frac{1}{2}} \partial y_{t} \partial_{t} \boldsymbol{H}\right\|_{L_{(D \times U)}^{2}}^{2}\right)(0)\right. \\
& \left.+\int_{0}^{t} C_{1}^{\epsilon \mu}\left(\left\|\epsilon^{\frac{1}{2}} \partial_{t}^{2} \boldsymbol{E}\right\|_{\boldsymbol{L}^{2}(D \times U)}^{2}+\left\|\mu^{\frac{1}{2}} \partial_{t}^{2} \boldsymbol{H}\right\|_{\left.\boldsymbol{L}_{(D \times U)}^{2}\right)}^{2}\right) d t\right] \\
& \leq \exp \left(t C_{1}^{\epsilon \mu}\right) \cdot\left[\left(\left\|\epsilon^{\frac{1}{2}} \partial_{y} \partial_{t} \boldsymbol{E}\right\|_{L^{2}(D \times U)}^{2}+\left\|\mu^{\frac{1}{2}} \partial y_{t} \partial_{t} \boldsymbol{H}\right\|_{L_{(D \times U)}^{2}}^{2}\right)(0)\right. \\
& \left.+t C_{1}^{\epsilon \mu}\left(\left\|\epsilon^{\frac{1}{2}} \partial_{t}^{2} \boldsymbol{E}\right\|_{L_{(D \times U)}^{2}}^{2}+\left\|\mu^{\frac{1}{2}} \partial_{t}^{2} \boldsymbol{H}\right\|_{L^{2}(D \times U)}^{2}\right)(0)\right],
\end{aligned}
$$

where in the last step we used Theorem 3.2 with $m=2$.

Substituting (3.14) into (3.13) and using the following estimates

$$
\int_{0}^{t} C_{1}^{\epsilon \mu} e^{t C_{1}^{\epsilon \mu}} d t=e^{t C_{1}^{\epsilon \mu}}-1 \leq e^{t C_{1}^{\epsilon \mu}}, \quad \int_{0}^{t} t C_{1}^{\epsilon \mu} e^{t C_{1}^{\epsilon \mu}} d t=t e^{t C_{1}^{\epsilon \mu}}-\frac{1}{C_{1}^{\epsilon \mu}}\left(e^{t C_{1}^{\epsilon \mu}}-1\right) \leq t e^{t C_{1}^{\epsilon \mu}},
$$

we conclude the proof. 


\subsection{The error estimate}

In this subsection, we are going to find out the error $G_{1}\left(\boldsymbol{E}(\cdot, \boldsymbol{y})-\boldsymbol{E}_{h}(\cdot, \boldsymbol{y})\right)$ and $G_{2}(\boldsymbol{H}(\cdot, \boldsymbol{y})-$ $\left.\boldsymbol{H}_{h}(\cdot, \boldsymbol{y})\right)$ where $\boldsymbol{y} \in U$ is given and $\boldsymbol{E}_{h}(\cdot, \boldsymbol{y})$ and $\boldsymbol{H}_{h}(\cdot, \boldsymbol{y})$ are the finite element solutions of (2.5)-(2.6).

Theorem 3.7. Under the same conditions as Theorem 3.1, we have the following error estimate

$$
\sqrt{\mathbb{E}\left[\left|I\left(G_{1}(\boldsymbol{E})\right)-Q_{N}\left(G_{1}\left(\boldsymbol{E}_{h}^{k}\right)\right)\right|^{2}+\left|I\left(G_{2}(\boldsymbol{H})\right)-Q_{N}\left(G_{2}\left(\boldsymbol{H}_{h}^{k}\right)\right)\right|^{2}\right]} \leq C\left(h^{r}+\tau^{2}+\varphi(M)^{-\frac{1}{2 \lambda}}\right) \text {. }
$$

Proof. By the triangle inequality, we have

$$
\mathbb{E}\left[\left|I\left(G_{1}(\boldsymbol{E})\right)-Q_{N}\left(G_{1}\left(\boldsymbol{E}_{h}^{k}\right)\right)\right|^{2}+\left|I\left(G_{2}(\boldsymbol{H})\right)-Q_{N}\left(G_{2}\left(\boldsymbol{H}_{h}^{k}\right)\right)\right|^{2}\right] \leq E r r_{1}+E r r_{2},
$$

where

$$
\begin{aligned}
& E r r_{1}=2 \mathbb{E}\left[\left|\left(I-Q_{N}\right)\left(G_{1}(\boldsymbol{E})\right)\right|^{2}+\left|\left(I-Q_{N}\right)\left(G_{2}(\boldsymbol{H})\right)\right|^{2}\right], \\
& E r r_{2}=2 \mathbb{E}\left[Q_{N}\left(G_{1}\left(\boldsymbol{E}-\boldsymbol{E}_{h}^{k}\right)\right)^{2}+Q_{N}\left(G_{2}\left(\boldsymbol{H}-\boldsymbol{H}_{h}^{k}\right)\right)^{2}\right] .
\end{aligned}
$$

The first term, which is the statistical error of QMC, can be estimated by Theorem 3.1. In fact, by Theorem 3.1 and the derivative estimates given by Theorems 3.4-3.5, we have

$$
\mathbb{E}\left[\left|\left(I-Q_{N}\right)\left(G_{1}(\boldsymbol{E})\right)\right|^{2}\right] \leq C \varphi(M)^{-\frac{1}{\lambda}}\|\boldsymbol{E}\|_{\mathcal{W}_{s, \gamma}} \leq C \varphi(M)^{-\frac{1}{\lambda}}
$$

Using the similar estimates for $\boldsymbol{H}$, we have

$$
\operatorname{Err}_{1} \leq C \varphi(M)^{-\frac{1}{\lambda}} .
$$

For the second term, we first notice that $G_{1}$ and $G_{2}$ are bounded on $L^{2}(D)$ :

$$
\left|G_{1}\left(\boldsymbol{E}-\boldsymbol{E}_{h}^{k}\right)\right| \leq\left\|G_{1}\right\|_{\left(\boldsymbol{L}^{2}(D)\right)^{*}}\left\|\boldsymbol{E}-\boldsymbol{E}_{h}^{k}\right\|_{\boldsymbol{L}^{2}(D)^{\prime}} \quad\left|G_{2}\left(\boldsymbol{H}-\boldsymbol{H}_{h}^{k}\right)\right| \leq\left\|G_{2}\right\|_{\left(\boldsymbol{L}^{2}(D)\right)^{*}}\left\|\boldsymbol{H}-\boldsymbol{H}_{h}^{k}\right\|_{\boldsymbol{L}^{2}(D)^{*}} .
$$

Applying the property that the QMC quadrature weights $1 / N$ are positive and have a sum 1 , we obtain

$$
\begin{aligned}
\mathbb{E}\left[Q_{N}\left(G_{1}\left(\boldsymbol{E}-\boldsymbol{E}_{h}^{k}\right)\right)^{2}\right] & \leq \mathbb{E}\left[Q_{N}\left(\left\|G_{1}\right\|_{\left(\boldsymbol{L}^{2}(D)\right)^{*}}\left\|\boldsymbol{E}-\boldsymbol{E}_{h}^{k}\right\|_{\boldsymbol{L}^{2}(D)}\right)^{2}\right] \\
& \leq C\left\|\boldsymbol{E}-\boldsymbol{E}_{h}^{k}\right\|_{\boldsymbol{L}^{2}(D)}^{2} \leq C\left(\tau^{2}+h^{r}\right)^{2} .
\end{aligned}
$$

Hence, by Theorem 2.1 we have

$$
E r r_{2} \leq C\left(\left\|\boldsymbol{E}-\boldsymbol{E}_{h}^{k}\right\|_{\boldsymbol{L}^{2}(D)}^{2}+\left\|\boldsymbol{H}-\boldsymbol{H}_{h}^{k}\right\|_{\boldsymbol{L}^{2}(D)}^{2}\right) \leq C\left(\tau^{2}+h^{r}\right)^{2} .
$$

Combining the estimates $E r r_{1}$ and $E r r_{2}$ together, we complete the proof. 


\section{Numerical experiments}

In this section, we present three numerical examples to verify our analysis. Note that the $3 \mathrm{D}$ theoretical analyses also hold true for $2 \mathrm{D}$ problems by interpreting the curl operators differently for $2 \mathrm{D}$ vectors and scalars, since for $2 \mathrm{D}$ problem one unknown is a vector and another unknown becomes a scalar. As shown in Section 2.1, we will apply the CrankNicolson scheme for the $T E_{z}$ mode, which has unknowns as electric field $\boldsymbol{E}:=\left(E_{x_{1}}, E_{x_{2}}\right)$ and magnetic field $H$, with the lowest order edge element on the triangular mesh. We will compute the sample means by using the single level Monte Carlo, multi-level Monte Carlo and QMC methods, respectively.

To test the convergence rate with an exact solution, we add additional source terms to the original governing equations. More specifically, we solve the following mixed finite element scheme: for any $k \geq 1$, find $E_{h}^{k}:=\left(E_{x_{1}, h}^{k}, E_{x_{2}, h}^{k}\right) \in V_{h}^{0}, H_{h}^{k} \in U_{h}$ such that

$$
\begin{aligned}
& \left(\epsilon \boldsymbol{E}_{h}^{k}, \boldsymbol{\phi}_{h}\right)-\frac{\tau}{2}\left(H_{h}^{k}, \nabla \times \boldsymbol{\phi}_{h}\right)=\left(\epsilon \boldsymbol{E}_{h}^{k-1}, \boldsymbol{\phi}_{h}\right)+\frac{\tau}{2}\left(H_{h}^{k-1}, \nabla \times \boldsymbol{\phi}_{h}\right)+\tau\left(f^{k-1 / 2}, \boldsymbol{\phi}_{h}\right), \\
& \left(\mu H_{h}^{k}, \psi_{h}\right)+\frac{\tau}{2}\left(\nabla \times \boldsymbol{E}_{h}^{k}, \psi_{h}\right)=\left(\mu H_{h}^{k-1}, \psi_{h}\right)-\frac{\tau}{2}\left(\nabla \times \boldsymbol{E}_{h}^{k-1}, \psi_{h}\right)+\tau\left(g^{k-1 / 2}, \psi_{h}\right)
\end{aligned}
$$

hold true for any $\boldsymbol{\phi}_{h} \in V_{h}^{0}$ and $\psi_{h} \in U_{h}$, where $f$ and $g$ are the added source terms. The finite element spaces $U_{h}$ and $V_{h}$ on a regular triangular mesh $\mathcal{T}_{h}$ of the domain $D=[0,1]^{2}$ are given as follows:

$$
\begin{aligned}
& U_{h}=\left\{\psi_{h} \in L^{2}(\Omega):\left.\psi_{h}\right|_{e} \text { is a constant, } \forall e \in \mathcal{T}_{h}\right\}, \\
& \boldsymbol{V}_{h}=\left\{\boldsymbol{\phi}_{h} \in H(\operatorname{curl} ; \Omega):\left.\boldsymbol{\phi}_{h}\right|_{e} \in \operatorname{span}\left\{\boldsymbol{\phi}_{i} \nabla \phi_{j}-\phi_{j} \nabla \phi_{i}\right\}, i, j=1,2,3, \forall e \in \mathcal{T}_{h}\right\},
\end{aligned}
$$

where $\phi_{i}$ denotes the barycentric coordinates of a triangular element $e$. To impose the 2D perfect conducting boundary condition, we introduce the subspace

$$
\boldsymbol{V}_{h}^{0}=\left\{\boldsymbol{\phi}_{h} \in \boldsymbol{V}_{h}: \hat{\tau} \cdot \phi_{h}=0, \text { on } \partial \Omega\right\},
$$

where $\hat{\tau}$ is the unit tangential vector on $\partial \Omega$.

\subsection{Single level Monte Carlo Method}

For this example, we adopt the following random coefficients and exact solutions: for any $t \in(0,1]$,

$$
\begin{aligned}
& \epsilon(x, y)=1+0.01\left(y_{1} x_{1}+y_{2} x_{2}+y_{3} x_{1}^{2}+y_{4} x_{2}^{2}+y_{5} x_{1} x_{2}+y_{6} x_{1}^{3}\right), \\
& \mu(x, y)=1+0.01\left(y_{1} x_{2}+y_{2} x_{1}+y_{3} x_{2}^{2}+y_{4} x_{1}^{2}+y_{5} x_{1} x_{2}+y_{6} x_{2}^{3}\right), \\
& E_{x}(x, y)=\sin \left(\pi x_{1}\right) \cos \left(\pi x_{2}\right) e^{-\pi t}(\epsilon+2 \mu), \\
& E_{y}(x, y)=-\cos \left(\pi x_{1}\right) \sin \left(\pi x_{2}\right) e^{-\pi t}(2 \epsilon+\mu), \\
& H(x, y)=\sin \left(\pi x_{1}\right) \sin \left(\pi x_{2}\right) e^{-\pi t}(\epsilon-2 \mu),
\end{aligned}
$$


Table 1: Errors of $\left(E_{x_{1}}, E_{x_{2}}, H\right)$ obtained by the single level Monte Carlo method with the lowest edge element.

\begin{tabular}{||c|c|c|c|c|c||}
\hline Mesh & $\left\|\mathbb{E}[\boldsymbol{E}(T)]-E_{M}\left[\boldsymbol{E}_{h}^{k}\right]\right\|_{l^{2}(D)}$ & Rate & $\left\|\mathbb{E}[H(T)]-E_{M}\left[H_{h}^{k}\right]\right\|_{l^{2}(D)}$ & Rate & CPU time(s) \\
\hline$N=2$ & $2.025681 E-01$ & - & $9.855452 E-01$ & - & 0.57096 \\
\hline$N=4$ & $8.016480 E-02$ & 1.9413 & $2.456558 E-01$ & 1.9868 & 13.29811 \\
\hline$N=8$ & $1.846385 E-02$ & 2.0869 & $6.260263 E-02$ & 1.9837 & 540.99794 \\
\hline$N=16$ & $4.241596 E-03$ & 2.0654 & $1.580314 E-02$ & 1.9891 & 43986.39742 \\
\hline$N=32$ & $1.053961 E-03$ & 2.0088 & $3.972438 E-03$ & 1.9921 & 258671.07102 \\
\hline
\end{tabular}

where $x=\left(x_{1}, x_{2}\right) \in D$ and $y=\left(y_{1}, \cdots, y_{6}\right) \in U=[0,1]^{6}$, i.e., $y_{i}$ are uniformly distributed random variables. The source functions $f$ and $g$ are obtained by plugging the exact solutions $E_{x}, E_{y}, H$ into the governing equations.

To test the convergence rate, we set the number of samples for Monte Carlo test as $M=$ $N^{4}$, where $N$ is the number of partition $D$ in both $x$ and $y$-direction, i.e., we first partition $D$ into $N \times N$ rectangles, then partition each rectangle into two triangles by connecting the diagonals. The total number of time steps is chosen as $N$ also. All the numerical tests have been carried out by using the FEniCS package (https:/ / fenicsproject.org/) on a 2017 MacBook Pro laptop with a $2.8 \mathrm{GHz}$ Intel Core i7 processor and a memory of $16 \mathrm{~GB}$ $2133 \mathrm{MHz}$ LPDDR3. The discrete $l^{2}(D)$ errors between the expectation of exact solution and the sample mean of numerical solution at the final time $T=1$ is computed to check our theoretical convergence rate given in Theorem 2.2.

Table 1 shows clearly that both the errors of $\boldsymbol{E}$ and $H$ are second order, which is due to the superconvergence phenomenon obtained for the lowest order triangular edge element [22]. Note that the finest mesh numerical test needs to solve the problem $32^{4}=1,048,576$ times, which takes about 72 hours, which shows that the simple level Monte Carlo method is unpractically slow. Later, we will show that the multi-level Monte Carlo and QMC methods are much more efficient than the simple level Monte Carlo method.

To further confirm our theoretical analysis, we resolve this example by using one order higher basis functions, i.e., a second order edge element for the electric field and linear Lagrange element for the magnetic field. The numerical results are presented in Table 2, which clearly shows the second order error estimate for both $E$ and $H$. This is consistent with Theorem 2.2.

\subsection{Multi-level Monte Carlo Method}

We repeat the last numerical example by using the multi-level Monte Carlo method analyzed in Section 2.3 and compute the sample mean by the telescope series of (2.26). At level $l$ of each numerical test, we set $h_{l}=\tau_{l}=1 / 2^{l}$ and $M_{l}=16^{L-l} l^{2}$, where $l=1, \cdots, L$. We 
Table 2: Errors of $\left(E_{x_{1}}, E_{x_{2}}, H\right)$ obtained by the single level Monte Carlo method with the second order edge element.

\begin{tabular}{||c|c|c|c|c|c||}
\hline Mesh & $\left\|\mathbb{E}[\boldsymbol{E}(T)]-E_{M}\left[\boldsymbol{E}_{h}^{k}\right]\right\|_{L^{2}(D)}$ & Rate & $\left\|\mathbb{E}[H(T)]-E_{M}\left[H_{h}^{k}\right]\right\|_{L^{2}(D)}$ & Rate & CPU time $(\mathrm{s})$ \\
\hline$N=2$ & $1.467381 E-01$ & - & $5.914026 E-01$ & - & 0.53130 \\
\hline$N=4$ & $3.563603 E-02$ & 2.0418 & $1.550685 E-01$ & 1.9312 & 15.71102 \\
\hline$N=8$ & $9.159853 E-03$ & 2.0009 & $3.790434 E-02$ & 1.9819 & 733.77024 \\
\hline$N=16$ & $2.232569 E-03$ & 2.0075 & $9.409349 E-03$ & 1.9954 & 67682.74943 \\
\hline
\end{tabular}

Table 3: Errors of $\left(E_{x_{1}}, E_{x_{2}}, H\right)$ obtained by the multi-level Monte Carlo method.

\begin{tabular}{||c|c|c|c|c|c||}
\hline Mesh & $\left\|\mathbb{E}[\boldsymbol{E}(T)]-E_{M}\left[E_{h}^{k}\right]\right\|_{l^{2}(D)}$ & Rate & $\left\|\mathbb{E}[H(T)]-E_{M}\left[H_{h}^{k}\right]\right\|_{l^{2}(D)}$ & Rate & CPU time $(\mathrm{s})$ \\
\hline$L=1$ & $8.022609 E-02$ & - & $2.464436 E-01$ & - & 0.61908 \\
\hline$L=2$ & $1.866771 E-02$ & 1.8555 & $6.106013 E-02$ & 2.0151 & 23.07685 \\
\hline$L=3$ & $4.516066 E-03$ & 1.7770 & $1.496582 E-02$ & 2.0165 & 437.75725 \\
\hline$L=4$ & $1.758842 E-03$ & 1.7113 & $3.842888 E-03$ & 2.0196 & 27501.86158 \\
\hline$L=5$ & $4.211790 E-04$ & 2.0621 & $9.102927 E-04$ & 2.0778 & 69150.66038 \\
\hline
\end{tabular}

choose the parameters to obtain $\mathcal{O}\left(\left(h_{L}\right)^{2}\right)$ convergence, since

$$
\sum_{l=1}^{L} h_{l}^{2} M_{l}^{-1 / 2}=\sum_{l=1}^{L} h_{L}^{2}\left(\frac{h_{l}}{h_{L}}\right)^{2} M_{l}^{-1 / 2}=h_{L}^{2} \sum_{l=1}^{L}\left(\frac{2^{L}}{2^{l}}\right)^{2}\left(16^{L-l} l^{2}\right)^{-1 / 2}=h_{L}^{2} \sum_{l=1}^{L} l^{-1}=\mathcal{O}\left(h_{L}^{2}\right) .
$$

As we can see from Table 3, the errors are still second order, which verifies Theorem 2.3. For the finest mesh case $(L=5)$, it requests total $\sum_{l=1}^{5} M_{l}=84,510$ Monte Carlo tests. Compared to the single level method, this saves a lot in the computational time as shown in Table 3.

\subsection{The QMC method}

This test is used to verify Theorem 3.7. We used the shifted lattice rule to generate the quasi random sequence on $[0,1]^{6}$. Since the theoretical convergent results of QMC in this case is of $\mathcal{O}\left(M^{-1+\epsilon_{m}}\right)$ where $0<\epsilon_{m} \ll 1$ (see [29]), we just take the total QMC test times $M=N^{2}$ for each test, where $N$ is the spatial and temporal partition number. As shown in Table 4, both the convergence rates of $E$ and $H$ are about second order. Note that the QMC sample for the finest temporal and spatial mesh is only $M=32^{2}=1,024$, which is much lower than both the single level and multi-level Monte Carlo methods. 
Table 4: Errors of $\left(E_{x_{1}}, E_{x_{2}}, H\right)$ obtained by the QMC method.

\begin{tabular}{||c|c|c|c|c|c||}
\hline Mesh & $\left\|\mathbb{E}[\boldsymbol{E}(T)]-E_{M}\left[E_{h}^{k}\right]\right\|_{L^{2}(D)}$ & Rate & $\left\|\mathbb{E}[H(T)]-E_{M}\left[H_{h}^{k}\right]\right\|_{L^{2}(D)}$ & Rate & CPU time(s) \\
\hline$N=2$ & $2.022678 E-01$ & - & $9.852111 E-01$ & - & 0.13250 \\
\hline$N=4$ & $8.019398 E-02$ & 1.9454 & $2.455848 E-01$ & 1.9872 & 0.71975 \\
\hline$N=8$ & $1.843664 E-02$ & 2.0936 & $6.260027 E-02$ & 1.9844 & 8.207584 \\
\hline$N=16$ & $4.244253 E-03$ & 2.0758 & $1.580382 E-02$ & 1.9903 & 165.89224 \\
\hline$N=32$ & $1.037406 E-03$ & 2.0325 & $3.965429 E-03$ & 1.9947 & 4970.95464 \\
\hline
\end{tabular}

\section{Conclusion}

In this paper, we have investigated the single level and multi-level Monte Carlo methods and the quasi-Monte Carlo method for solving the time-dependent Maxwell's equations with random inputs. The convergences of all methods have been established. Note that the QMC method's convergence rate depends on the regularity or smoothness of the random inputs. Numerical results supporting the analysis are presented. As for future work, we plan to consider higher order QMC rules such as the multi-level QMC, and applications to practical uncertainty quantification problems for Maxwell's equations.

\section{Acknowledgments}

The authors would like to thank the referees for their careful reading and kind suggestions for improving the paper, and this work was partially supported by National Natural Science Foundation of China under grants No. 11961048 and No. 11671340, and NSF of Jiangxi Province with No. 20181ACB20001.

\section{References}

[1] I.M. Babuska, F. Nobile and R. Tempone, A stochastic collocation method for elliptic partial differential equations with random input data, SIAM J. Numer. Anal. 435 (2007) 1005-1034.

[2] I. Babuska, R. Tempone and G. Zouraris, Galerkin finite element approximations of stochastic elliptic differential equations, SIAM J. Numer. Anal. 42 (2004) 800-825.

[3] G. Bao, C. Chen and P. Li, Inverse random source scattering for elastic waves, SIAM J. Numer. Anal. 55(6) (2017) 2616-2643.

[4] A. Barth, C. Schwab and N. Zollinger, Multi-level Monte Carlo finite element method for elliptic PDEs with stochastic coefficients. Numer. Math. 119(1) (2011) 123-161.

[5] P. Benner and J. Schneider, Uncertainty quantification for Maxwell's equations using stochastic collocation and model order reduction, Int. J. Uncertain. Quantif. 5(3) (2015) 195208.

[6] C. Chauviére, J.S. Hesthaven and L. Lurati, Computational modeling of uncertainty in timedomain electromagnetics, SIAM J. Sci. Comput. 28(2) (2006) 751-775. 
[7] C. Chen, J. Hong and L. Ji, Mean-square convergence of a semidiscrete scheme for stochastic Maxwell equations, SIAM J. Numer. Anal. 57(2) (2019) 728-750.

[8] P. Chen, A. Quarteroni and G. Rozza, A weighted reduced basis method for elliptic partial differential equations with random input data, SIAM J. Numer. Anal. 51 (2013) 3163-3185.

[9] K.A. Cliffe, M.B. Giles, R. Scheichl and A.L. Teckentrup, Multilevel Monte Carlo methods and applications to elliptic PDEs with random coefficients, Comput. Vis. Sci. 14(1) (2011) 3-15.

[10] R. Cools, F. Y. Kuo and D. Nuyens, Constructing embedded lattice rules for multivariable integration, SIAM J. Sci. Comput. 28(6) (2006) 2162-2188.

[11] J. Dick, F.Y. Kuo and I.H. Sloan, High-dimensional integration: the quasi-Monte Carlo way, Acta Numer. 22 (2013) 133-288.

[12] J. Dick, I.H. Sloan, X. Wang and H. Woźniakowski, Liberating the weights, J. Complexity, 20 (2004) 593-623.

[13] P. Dostert, Y. Efendiev, and T.Y. Hou, Multiscale finite element methods for stochastic porous media flow equations and application to uncertainty quantification, Comput. Methods Appl. Mech. Engrg. 197 (2008) 3445-3455.

[14] Z. Fang, J. Li, T. Tang and T. Zhou, Efficient stochastic Galerkin methods for Maxwell's equations with random inputs, J. Sci. Comput. 80 (2019) 248-267.

[15] X. Feng and C. Lorton, An efficient Monte Carlo interior penalty discontinuous Galerkin method for elastic wave scattering in random media, Comput. Methods Appl. Mech. Engrg. 315 (2017) 141-168.

[16] R. Ghanem and P. Spanos, Stochastic Finite Elements: A Spectral Approach, Springer, New York, 1991.

[17] M.B. Giles, Multilevel Monte Carlo methods, Acta Numer. 2015 259-328.

[18] I.G. Graham, F.Y. Kuo, J.A. Nichols, R. Scheichl, Ch. Schwab and I.H. Sloan, Quasi-Monte Carlo finite element methods for elliptic PDEs with lognormal random coefficients, Numer. Math. 131 (2015) 329-368.

[19] M.D. Gunzburger, C.G. Webster and G. Zhang, Stochastic finite element methods for partial differential equations with random input data, Acta Numer. 23 (2014) 521-650.

[20] Y. Hao, F. Kang, J. Li and K. Zhang, Computation of moments for Maxwell's equations with random interfaces via pivoted low-rank approximation, J. Comput. Phys. 371 (2018) 1-19.

[21] H. Harbrecht, M. Peters and M. Siebenmorgen, Multilevel accelerated quadrature for PDEs with log-normally distributed diffusion coefficient, SIAM/ASA J. Uncertain. Quantif. 4(1) (2016) 520-551.

[22] Y. Huang, J. Li and C. Wu, Averaging for superconvergence: Verification and application of 2D edge elements to Maxwell's equations in metamaterials, Comput. Methods Appl. Mech. Engrg. 255 (2013) 121-132.

[23] C. Jerez-Hanckes, C. Schwab and J. Zech, Electromagnetic wave scattering by random surfaces: shape holomorphy, Math. Models Methods Appl. Sci. 27(12) (2017) 2229-2259.

[24] S. Jin, D. Xiu and X.Zhu, A well-balanced stochastic galerkin method for scalar hyperbolic balance laws with random inputs, J. Sci. Comput. 67(3) (2016) 1198-1218.

[25] D. Kamilis and N. Polydorides, Uncertainty quantification for low-frequency, timeharmonic Maxwell equations with stochastic conductivity models, SIAM/ASA J. Uncertainty Quantification 6-4 (2018) 1295-1334.

[26] M. Kovacs, S. Larsson and F. Saedpanah, Finite element approximation of the linear stochastic wave equation with additive noise, SIAM J. Numer. Anal. 48 (2010) 408-427.

[27] F.Y. Kuo and D. Nuyens, Application of quasi-Monte Carlo methods to elliptic PDEs with 
random diffusion coefficients: a survey of analysis and implementation, Found Comput. Math. 16 (2016) 1631-1696.

[28] F.Y. Kuo, C. Schwab and I.H. Sloan, Quasi-Monte Carlo methods for high-dimensional integration: the standard (weighted Hilbert space) setting and beyond, The ANZIAM Journal 53(1) (2011) 1-37.

[29] F.Y. Kuo, C. Schwab and I.H. Sloan, Quasi-Monte Carlo finite element methods for a class of elliptic partial differential equations with random coefficients, SIAM J. Numer. Anal. 50 (2012) 3351-3374.

[30] J. Li and Z. Fang, Analysis and application of stochastic collocation methods for Maxwell's equations with random inputs, Adv. Appl. Math. Mech. 10(6) (2018) 1305-1326.

[31] J. Li, Z. Fang and G. Lin, Regularity analysis of metamaterial Maxwell's equations with random coefficients and initial conditions, Comput. Methods Appl. Mech. Engrg. 335 (2018) 24-51.

[32] J. Li and Y. Huang, Time-Domain Finite Element Methods for Maxwell's Equations in Metamaterials, Springer Series in Computational Mathematics, vol.43, Springer, 2013.

[33] G. Lord, C.E. Powell and T. Shardlow, An introduction to computational stochastic PDEs, Cambridge University Press, 2014.

[34] P. Monk, Finite Element Methods for Maxwell's Equations, Oxford University Press, Oxford, 2003.

[35] M. Motamed, F. Nobile and R. Tempone, A stochastic collocation method for the second order wave equation with a discontinuous random speed, Numer. Math. 123 (2013) 493-536.

[36] A. Narayan and T. Zhou, Stochastic collocation methods on unstructured meshes, Commun. Comput. Phys. 18 (2015) 1-36.

[37] U. Römer, S. Schöps and T. Weiland, Stochastic modeling and regularity of the nonlinear elliptic curl-curl equation, SIAM/ ASA J. Uncertainty Quantification 4 (2016) 952-979.

[38] C. Schwab and C.J. Gittelson, Sparse tensor discretizations of high-dimensional parametric and stochastic PDEs, Acta Numer. 20 (2011) 291-467.

[39] I.H. Sloan, X. Wang and H. Woźniakowski, Finite-order weights imply tractability of multivariate integration, J. Complexity 20 (2004) 46-74.

[40] I.H. Sloan and H. Woźniakowski, When are quasi-Monte Carlo algorithms efficient for highdimensional integrals? J. Complexity 14 (1998) 1-33.

[41] R.C. Smith, Uncertainty Quantification: Theory, Implementation, and Applications, SIAM, 2014.

[42] T.J. Sullivan, Introduction to Uncertainty Quantification, Springer, 2015.

[43] T. Tang and T. Zhou, Convergence analysis for stochastic collocation methods to scalar hyperbolic equations with random wave speed, Commun. Comput. Phys. 8(1) (2010) 226-248.

[44] A.L. Teckentrup, R. Scheichl, M.B. Giles and E. Ullmann, Further analysis of multilevel Monte Carlo methods for elliptic PDEs with random coefficient, Numer. Math. 125 (2013) 569-600.

[45] J. Tryoen, O. LeMaitre, M. Ndjinga and A. Ern, Intrusive Galerkin methods with upwinding for uncertain nonlinear hyperbolic systems, J. Comput. Phys. 229 (2010) 6485-6511.

[46] I.C. Tsantili, M.H. Cho, W. Cai and G. EM Karniadakis, A computational stochastic methodology for the design of random meta-materials under geometric constraints, SIAM J. Sci. Comput. 40(2) (2018) B353-B378.

[47] D. Xiu, Numerical Methods for Stochastic Computations: A Spectral Method Approach, Princeton University Press, 2010.

[48] D. Xiu and J. Shen, Efficient stochastic galerkin methods for random diffusion equations, J. 
Comput. Phys. 228(2) (2009) 266-281.

[49] Z. Zhang, X. Hu, T.Y. Hou, G. Lin and M. Yan, An adaptive ANOVA-based data-driven stochastic method for elliptic PDEs with random coefficient, Commun. Comput. Phys. 16(3) (2014) 571-598.

[50] Z. Zhang and G.E. Karniadakis, Numerical Methods for Stochastic Partial Differential Equations with White Noise, Springer, 2017.

[51] X. Zhong and Q. Li, Galerkin methods for stationary radiative transfer equations with uncertain coefficients, J. Sci. Comput. 76 (2018) 1105-1126.

[52] T. Zhou and T. Tang, Galerkin methods for stochastic hyperbolic problems using biorthogonal polynomials, J. Sci. Comput. 51 (2012) 274-292. 